\title{
Adaptive Kalman Estimation in Target Tracking Mixed with Random One-Step Delays, Stochastic-Bias Measurements, and Missing Measurements
}

\author{
Sujuan Chen, Yinya Li, Guoqing Qi, and Andong Sheng \\ School of Automation, Nanjing University of Science and Technology, Nanjing 210094, China \\ Correspondence should be addressed to Sujuan Chen; chensujuan2010@126.com
}

Received 5 November 2013; Accepted 21 November 2013

Academic Editor: Lifeng Ma

Copyright (c) 2013 Sujuan Chen et al. This is an open access article distributed under the Creative Commons Attribution License, which permits unrestricted use, distribution, and reproduction in any medium, provided the original work is properly cited.

\begin{abstract}
The objective of this paper is concerned with the estimation problem for linear discrete-time stochastic systems with mixed uncertainties involving random one-step sensor delay, stochastic-bias measurements, and missing measurements. Three Bernoulli distributed random variables are employed to describe the uncertainties. All the three uncertainties in the measurement have certain probability of occurrence in the target tracking system. And then, an adaptive Kalman estimation is proposed to deal with this problem. The adaptive filter gains can be obtained in terms of solutions to a set of recursive discrete-time Riccati equations. Examples in three scenarios of target tracking are exploited to show the effectiveness of the proposed design approach.
\end{abstract}

\section{Introduction}

In practical target tracking, the missing phenomenon of the sensor measurement often occurs. For example, the measurement value may contain noise only due to the shelter of the obstacles, a high noise environment, a failure in the measurement, intermittent sensor failures, high maneuverability of tracked target, and so forth. Thus, the estimation for systems with missing measurements has received much attention during the few years. In general, there are two ways to describe the missing phenomenon. One way is to model the uncertainty by using a stochastic Bernoulli binary switching sequence taking on values of 0 and 1 (see, e.g., [1-18] and the references therein). The suboptimal filtering algorithm [2] in the minimum variance sense with only missing measurements has been proposed and the robust filter [14] is designed. In [3] Sinopoli et al. studied the statistical behavior of the Kalman filter error covariance with varying Kalman gain with missing measurements and the existence of a critical value has been shown for the arrival rate of the observations. The stochastic stability of the extended kalman filter with missing measurements is analyzed in [8], while stochastic stability of the unscented kalman filter with missing measurements is studied in [13]. For benchmarking the performance of any estimation algorithm with missing measurements in advance, the modified Cramer-Rao bound (CRLB) and modified Riccati equation have been studied in $[1,15-18]$. Another way is to model the uncertainty as a Markovian jumping sequence (see [19-21] and the references therein). The convergence for semi-Markov chains has been studied in [20], where the exponential filter is designed in [21].

For the case of random delayed measurements, the delays will cause performance degradation or instability with traditional Kalman filters. Therefore, a large volume of literature has been published on the topic of filtering problem with delayed measurements; see, for example, $[6,7,10-12,22-$ 27]. Most of the publications assume that the delayed measurements are always deterministic. However, it is not often the case in actual target tracking, where the time delays in the measurement may occur in a random way. A stochastic Bernoulli binary switching sequence taking on values of 0 and 1 has been employed to describe the randomly delayed measurements. In such case, the minimum variance suboptimal filter $[22,26]$ and the robust filter with only delayed measurements [25] are obtained.

For the case of stochastic-bias measurements, a twostage kalman estimator for state estimation in the presence 
of random bias and for tracking maneuvering targets is given in [28], while an optimal two-stage kalman filter in the presence of random bias is obtained in [29]. Moreover, optimal and suboptimal separate-bias kalman estimators for a stochastic bias are studied in [30]. The biased bearingonly parameter estimation for bistatic system is studied in [31] and the Cramer-Rao lower bound for biased bearingsonly maneuvering target tracking is obtained in [32], where the stochastic-bias phenomenon is described by a stochastic Bernoulli binary switching sequence taking on values of 0 and 1.

Based on the stochastic parameterized description, recently, some suboptimal and optimal filtering algorithms have been proposed for systems with both sensor delays and missing measurements [10-12, 21, 24, 33, 34]. Adaptive kalman filtering [10] is designed mixed with random sensor delays, multiple packet dropouts, and missing measurements. The problem of robust state estimation for two-dimensional stochastic time-delay systems [12] is studied with missing measurements and sensor saturation. An optimal linear filter for discrete-time systems with randomly delayed and lost measurements with/without time stamps is studied in [34]. Up to now, the state estimation problem for systems in target tracking mixed with random one-step delays, stochasticbias measurements, and missing measurements is seldom reported, which almost exist simultaneously in target tracking. Motivated by the results above, a new mathematical model is developed to describe the random one-step delays, stochastic-bias measurements, and missing measurements using three Bernoulli distributed random variables taking on values of 0 and 1 . By state augmentation, it is converted to a stochastic parameterized system. Based on the model, An adaptive kalman estimation is obtained in target tracking via projection theory. The rest of the paper is organized as follows. Section 2 establishes the new system model and introduces the augment state Kalman estimation to deal with the proposed problem. Section 3 presents an adaptive estimation algorithm. In Section 4, simulation results are analyzed and are used to evaluate the proposed algorithm. A summary of our conclusions is given in Section 5 .

\section{System Model and Problem Formulation}

Consider the following stochastic-biased discrete time linear time-varying state-space system with missing measurements:

$$
\begin{gathered}
X_{k+1}=F_{k} X_{k}+w_{k}, \quad X_{k}(k \leq 0)=X_{0}, \\
y_{k}=d_{k} H_{k} X_{k}+d_{k}\left(1-l_{k}\right) \tau_{k}+v_{k},
\end{gathered}
$$

where $X_{k} \in \mathbb{R}^{n}$ is the state vector, $y_{k} \in \mathbb{R}^{m}$ is the measured output, and $w_{k} \in \mathbb{R}^{p}$ and $v_{k} \in \mathbb{R}^{m}$ are Gaussian random vectors with zero mean and covariance matrices $\sigma_{w}^{2} \geq 0$ and $\sigma_{v}^{2} \geq 0$, respectively. The bias vector $\tau_{k} \in \mathbb{R}^{m}$ is treated as a zero mean Gaussian random vector with a priori known covariance $\sigma_{\tau}^{2} . F_{k}$ and $H_{k}$ are constant matrices with compatible dimensions. The $d_{k}$ is a scalar binary Bernoulli distributed random variable taking values $\{1,0\}$ which are defined as $\operatorname{Prob}\left\{d_{k}=1\right\}=\lambda$ and $\operatorname{Prob}\left\{d_{k}=0\right\}=1-\lambda$.
If $d_{k}=1$, the measurement at time $k$ is available; otherwise, the measurement is missing; that is, the received observation is only the measurement noise at time $k$. The $l_{k}$ is a scalar binary Bernoulli distributed random variable taking values $\{1,0\}$ which are defined as $\operatorname{Prob}\left\{l_{k}=1\right\}=\eta$ and $\operatorname{Prob}\left\{l_{k}=\right.$ $0\}=1-\eta$. If $d_{k}=1$ and $l_{k}=0$, the measurement at time is available and unbiased; else if $d_{k}=1$ and $l_{k}=1$, the measurement at time $k$ is available but biased.

Assume that the initial state $X_{0}$ satisfies the mean and covariance conditions:

$$
E\left\{X_{0}\right\}=\mu_{0}, \quad E\left\{\left(X_{0}-\mu_{0}\right)\left(X_{0}-\mu_{0}\right)^{\mathrm{T}}\right\}=P_{0},
$$

where $E\{\cdot\}$ is the mathematical expectation operator and the superscript $\mathrm{T}$ is the transpose. The initial state $\mu_{0}$ is uncorrelated with $w_{k}, v_{k}$, and $\tau_{k}$.

Next, we will establish a unified model to describe the mixed uncertainties of missing measurements, one-step delay, and stochastic biased measurements by Bernoulli distributed random variables.

Define

$$
\underline{X}_{k+1}=\left[\begin{array}{lll}
X_{k+1}^{\mathrm{T}}, & X_{k}^{\mathrm{T}}, & y_{k}^{\mathrm{T}}
\end{array}\right]^{\mathrm{T}} \text {. }
$$

A compact representation of the system (1) with mixed uncertainties of missing measurements, one-step delay, and stochastic-biased measurements can be given in an augmented form as follows:

$$
\begin{gathered}
\underline{X}_{k+1}=A_{k} \underline{X}_{k}+B_{k} W_{k}+M_{k} \Lambda_{k}, \\
y_{k}=C_{k} \underline{X}_{k}+D_{k} W_{k}+N_{k} \Lambda_{k},
\end{gathered}
$$

where

$$
\begin{aligned}
& W_{k}=\left[\begin{array}{lll}
w_{k}^{\mathrm{T}}, & v_{k}^{\mathrm{T}}, & v_{k-1}^{\mathrm{T}}
\end{array}\right]^{\mathrm{T}} \text {, } \\
& \Lambda_{k}=\left[\tau_{k}^{\mathrm{T}}, \tau_{k-1}^{\mathrm{T}}\right]^{\mathrm{T}} \text {. }
\end{aligned}
$$

Further, we have the following statistical information about $W_{k}$ and $\Lambda_{k}$ :

$$
\begin{gathered}
E\left\{W_{k}\right\}=0, \\
E\left\{\Lambda_{k}\right\}=0, \\
P_{W_{k}}=E\left\{W_{k} W_{k}^{\mathrm{T}}\right\}=\operatorname{diag}\left\{\sigma_{w}^{2}, \sigma_{v}^{2}, \sigma_{v}^{2}\right\}, \\
E\left\{v_{k} v_{r}^{\mathrm{T}}\right\}=0, \quad k \neq r, \\
P_{\Lambda_{k}}=E\left\{\Lambda_{k} \Lambda_{k}^{\mathrm{T}}\right\}=\operatorname{diag}\left\{\sigma_{\tau}^{2}, \sigma_{\tau}^{2}\right\}, \\
E\left\{\tau_{k} \tau_{r}^{\mathrm{T}}\right\}=0, \quad k \neq r .
\end{gathered}
$$

Let $\left\{A_{k}^{q}, B_{k}^{q}, C_{k}^{q}, D_{k}^{q}, M_{k}^{q}, N_{k}^{q} \mid q=1,2,3\right\}$ denote the three models corresponding, respectively, to systems with no missing measurement, one-step sensor delay, and missing 
measurement. They are defined by the following system matrices:

(a) current measurement case:

$$
\begin{gathered}
A_{k}^{1}=\left[\begin{array}{ccc}
F_{k} & 0 & 0 \\
I & 0 & 0 \\
H_{k} & 0 & 0
\end{array}\right], \quad B_{k}^{1}=\left[\begin{array}{lll}
I & 0 & 0 \\
0 & 0 & 0 \\
0 & I & 0
\end{array}\right], \\
M_{k}^{1}=\left(1-l_{k}\right)\left[\begin{array}{ll}
0 & 0 \\
0 & 0 \\
I & 0
\end{array}\right]=\left(1-l_{k}\right) \underline{M}_{k}^{1}, \quad C_{k}^{1}=\left[\begin{array}{lll}
H_{k} & 0 & 0
\end{array}\right], \\
D_{k}^{1}=\left[\begin{array}{lll}
0 & I & 0
\end{array}\right], \quad N_{k}^{1}=\left(1-l_{k}\right)\left[\begin{array}{ll}
I & 0
\end{array}\right]=\left(1-l_{k}\right) \underline{N}_{k}^{1},
\end{gathered}
$$

(b) one-step random sensor delay case:

$$
\begin{gathered}
A_{k}^{2}=\left[\begin{array}{ccc}
F_{k} & 0 & 0 \\
I & 0 & 0 \\
0 & H_{k} & 0
\end{array}\right], \quad B_{k}^{2}=\left[\begin{array}{lll}
I & 0 & 0 \\
0 & 0 & 0 \\
0 & 0 & I
\end{array}\right], \\
M_{k}^{2}=\left(1-l_{k}\right)\left[\begin{array}{ll}
0 & 0 \\
0 & 0 \\
0 & I
\end{array}\right]=\left(1-l_{k}\right) M_{k}^{2}, \\
C_{k}^{2}=\left[\begin{array}{ll}
0 & H_{k-1}
\end{array}\right], \\
D_{k}^{2}=\left[\begin{array}{lll}
0 & 0 & I
\end{array}\right], \quad N_{k}^{2}=\left(1-l_{k}\right)\left[\begin{array}{ll}
0 & I
\end{array}\right]=\left(1-l_{k}\right) \underline{N}_{k}^{2},
\end{gathered}
$$

(c) missing measurement case:

$$
\begin{gathered}
A_{k}^{3}=\left[\begin{array}{ccc}
F_{k} & 0 & 0 \\
I & 0 & 0 \\
0 & 0 & 0
\end{array}\right], \quad B_{k}^{3}=\left[\begin{array}{lll}
I & 0 & 0 \\
0 & 0 & 0 \\
0 & I & 0
\end{array}\right], \\
M_{k}^{3}=\left(1-l_{k}\right)\left[\begin{array}{ll}
0 & 0 \\
0 & 0 \\
0 & 0
\end{array}\right]=\left(1-l_{k}\right) \underline{M}_{k}^{3}, \\
C_{k}^{3}=\left[\begin{array}{lll}
0 & 0 & 0
\end{array}\right], \quad D_{k}^{3}=\left[\begin{array}{lll}
0 & 0 & 0
\end{array}\right], \\
N_{k}^{3}=\left(1-l_{k}\right)\left[\begin{array}{ll}
0 & 0
\end{array}\right]=\left(1-l_{k}\right) \underline{N}_{k}^{3} .
\end{gathered}
$$

The systems $\left\{A_{k}, B_{k}, C_{k}, D_{k}, M_{k}, N_{k}\right\}$ can be represented as follows:

$$
\left\{A_{k}, B_{k}, C_{k}, D_{k}, M_{k}, N_{k}\right\}:=\sum_{q=1}^{3} \beta_{q}\left\{A_{k}^{q}, B_{k}^{q}, C_{k}^{q}, D_{k}^{q}, M_{k}^{q}, N_{k}^{q}\right\},
$$

where $\beta_{q}$ satisfies

$$
\sum_{q=1}^{3} \beta_{q}=1, \quad \beta_{q}=0 \text { or } 1 .
$$

Assume that we are able to model the real system at time $k$ by analyzing the system. The probability of the system $\left\{A_{k}^{q}, B_{k}^{q}, C_{k}^{q}, D_{k}^{q}, M_{k}^{q}, N_{k}^{q} \mid q=1,2,3\right\}$ at time $k$ is $\rho_{q}$. Obviously, we have $\sum_{q=1}^{3} \rho_{q}=1$.
Remark 1. In this paper, the uncertain model subject to missing measurements, one-step delay, and stochastic-biased measurements is considered; that is, the probabilities of the stochastic uncertainties are known. Here the missing measurements are replaced by zeros; that is, the measurement containing only noise is not a valid value. We can examine the "error detection" bits before sending them to the estimator. The delay may be much longer and the receiver (estimator side) cannot know the values without time stamps. Therefore, we cannot have an exact model $\left\{A_{k}^{q}, B_{k}^{q}, C_{k}^{q}, D_{k}^{q}, M_{k}^{q}, N_{k}^{q}\right.$ । $q=1,2,3\}$; that is, the value of $q$ is unknown when the measurement arrives in an estimator. In this case, we need to know the probability $\rho_{q}(q=1,2,3)$ of each model $\left\{A_{k}^{q}, B_{k}^{q}\right.$, $\left.C_{k}^{q}, D_{k}^{q}, M_{k}^{q}, N_{k}^{q} \mid q=1,2,3\right\}$

Define

$$
\underline{X}_{k+1}=E\left\{\left[X_{k+1}^{\mathrm{T}}, X_{k}^{\mathrm{T}}\right]^{\mathrm{T}}\right\} \text {. }
$$

That is, $\underline{X}_{k+1}$ is an estimator vector of $\left[X_{k+1}^{\mathrm{T}}, X_{k}^{\mathrm{T}}\right]^{\mathrm{T}}$.

We can construct an estimator of the following structure:

$$
\underline{X}_{k+1}=\widehat{A}_{k} \widehat{X}_{k}+G_{k}\left(y_{k}-\widehat{C}_{k}^{q} \widehat{X}_{k}\right) .
$$

Define

$$
S=\left[\begin{array}{ll}
I & 0
\end{array}\right]
$$

From (12), we know that $\underline{X}_{k+1}$ is an estimate vector for $\left[\begin{array}{ll}X_{k+1}^{\mathrm{T}}, & X_{k}^{\mathrm{T}}\end{array}\right]^{\mathrm{T}}$. Therefore, it is obvious that we can choose $\underline{A}_{k}$ and $\widehat{C}_{k}^{q}$ as follows:

$$
\widehat{A}_{k}=\left[\begin{array}{cc}
F_{k} & 0 \\
I & 0
\end{array}\right], \quad \widehat{C}_{k}^{q}=C_{k}^{q} S^{\mathrm{T}}
$$

The state estimation error covariance is defined by

$$
P_{e_{k}}=E\left\{e_{k} e_{k}^{\mathrm{T}}\right\}
$$

where

$$
e_{k}=X_{k}-\widehat{X}_{k}
$$

In order to obtain the optimal $G_{k}$, we can minimize $P_{e_{k}}$.

\section{Estimation Algorithm Mixed with One-Step Sensor Delays, Missing Measurements, and Stochastic-Biased Measurements}

In this section, based on the stochastic system models $\left\{A_{k}^{q}, B_{k}^{q}, C_{k}^{q}, D_{k}^{q}, M_{k}^{q}, N_{k}^{q} \mid q=1,2,3\right\}$, we will derive the optimal $G_{k}$ before giving one-step predictions.

From (3) and (14), we can readily have that

$$
S \underline{X}_{k}=\left[\begin{array}{ll}
X_{k}^{\mathrm{T}}, & X_{k-1}^{\mathrm{T}}
\end{array}\right]^{\mathrm{T}} .
$$

Define

$$
\xi_{k+1}=S \underline{X}_{k+1}-\underline{X}_{k+1}
$$


Substituting (4) and (13) into (19) yields

$$
\begin{aligned}
\xi_{k+1}=\sum_{q=1}^{3} \beta_{q}\{ & S A_{k}^{q} \underline{X}_{k}+\left(\widehat{A}_{k}-G_{k} \widehat{C}_{k}^{q}\right) \underline{X}_{k} \\
& +\left(S B_{k}^{q}-G_{k} D_{k}^{q}\right) W_{k}+\left(S M_{k}^{q}-G_{k} N_{k}^{q}\right) \Lambda_{k} \\
& \left.-G_{k} C_{k}^{q} \underline{X}_{k}\right\} .
\end{aligned}
$$

Observing (7)-(10) and (14), we have the following results:

$$
\begin{gathered}
S A_{k}^{q} \underline{X}_{k}=\widehat{A}_{k} S \underline{X}_{k}, \\
C_{k}^{q} \underline{X}_{k}=C_{k}^{q} S^{\mathrm{T}} S \underline{X}_{k}, \\
C_{k}^{q} \underline{X}_{k}=\widehat{C}_{k}^{q} S \underline{X}_{k} .
\end{gathered}
$$

Furthermore, substituting (21) into (20), we can obtain the following equation:

$$
\begin{aligned}
\xi_{k+1}=\sum_{q=1}^{3} \beta_{q}\{ & \left(\widehat{A}_{k}-G_{k} \widehat{C}_{k}^{q}\right) \xi_{k} \\
& +\left(S B_{k}^{q}-G_{k} D_{k}^{q}\right) W_{k}+\left(S M_{k}^{q}-G_{k} N_{k}^{q}\right) \Lambda_{k} \\
& \left.+G_{k}\left(C_{k}^{q} S^{T}-\widehat{C}_{k}^{q}\right) S \underline{X}_{k}\right\} .
\end{aligned}
$$

Noting that $\widehat{C}_{k}^{q}=C_{k}^{q} S^{\mathrm{T}},(22)$ can be rewritten as

$$
\begin{aligned}
\xi_{k+1}=\sum_{q=1}^{3} \beta_{q}\left\{\left(\widehat{A}_{k}-G_{k} \widehat{C}_{k}^{q}\right) \xi_{k}+\left(S B_{k}^{q}-G_{k} D_{k}^{q}\right) W_{k}\right. \\
\left.+\left(S M_{k}^{q}-G_{k} N_{k}^{q}\right) \Lambda_{k}\right\} .
\end{aligned}
$$

Using the statistics of $W_{k}$ and $\Lambda_{k}$ in (6), the covariance matrix of $\xi_{k}$ can be computed by

$$
\begin{aligned}
P_{\xi_{k+1}}=\sum_{q=1}^{3} \beta_{q}\{ & \left(\widehat{A}_{k}-G_{k} \widehat{C}_{k}^{q}\right) P_{\xi_{k}}\left(\widehat{A}_{k}-G_{k} \widehat{C}_{k}^{q}\right)^{\mathrm{T}} \\
& +\left(S B_{k}^{q}-G_{k} D_{k}^{q}\right) P_{W_{k}}\left(S B_{k}^{q}-G_{k} D_{k}^{q}\right)^{\mathrm{T}} \\
& +(1-\eta)\left(S \underline{M}_{k}^{q}-G_{k} \underline{N}_{k}^{q}\right) \\
& \left.\times P_{\Lambda_{k}}\left(S \underline{M}_{k}^{q}-G_{k} \underline{N}_{k}^{q}\right)^{\mathrm{T}}\right\} .
\end{aligned}
$$

From (17) and (19), we can easily have

$$
e_{k+1}=\Phi \xi_{k+1}, \quad \Phi=\left[\begin{array}{ll}
I & 0
\end{array}\right] .
$$

Therefore, the problem of minimizing $P_{e_{k}}$ is equivalent to

$$
\min _{G_{k}} E\left\{\operatorname{Tr}\left\{\Phi P_{\xi_{k+1}} \Phi^{\mathrm{T}}\right\}\right\} \quad \text { subject to }(24) .
$$

Let $K_{k}=\Phi G_{k}$ and left- and right-multiply (24) by $\Phi$ and $\Phi^{\mathrm{T}}$, respectively. The estimation error covariance matrix can be computed by

$$
\begin{aligned}
P_{e_{k+1}}= & \sum_{q=1}^{3} \beta_{q}\left\{\left(\Phi \widehat{A}_{k}-K_{k} \widehat{C}_{k}^{q}\right) P_{\xi_{k+1}}\left(\Phi \widehat{A}_{k}-K_{k} \widehat{C}_{k}^{q}\right)^{\mathrm{T}}\right\} \\
& +\sum_{q=1}^{3} \beta_{q}\left\{\left(\Phi S B_{k}^{q}-K_{k} D_{k}^{q}\right) P_{W_{k}}\left(\Phi S B_{k}^{q}-K_{k} D_{k}^{q}\right)^{\mathrm{T}}\right\}
\end{aligned}
$$

$$
\begin{aligned}
+\sum_{q=1}^{3} \beta_{q}\{ & (1-\eta)\left(\Phi S \underline{M}_{k}^{q}-K_{k} \underline{N}_{k}^{q}\right) \\
& \left.\times P_{\Lambda_{k}}\left(\Phi S \underline{M}_{k}^{q}-K_{k} \underline{N}_{k}^{q}\right)^{\mathrm{T}}\right\} .
\end{aligned}
$$

Differentiate $E\left\{\operatorname{Tr} P_{e_{k+1}}\right\}$ with respect to $K_{k}$ and set the derivative to zero. We can obtain the optimal $K_{k}$ as follows:

$$
\begin{aligned}
K_{k}= & {\left[\sum_{q=1}^{3} \beta_{q} \Phi \widehat{A}_{k} P_{\xi_{k+1}}\left(\widehat{C}_{k}^{q}\right)^{\mathrm{T}}+\sum_{q=1}^{3} \beta_{q} \Phi S B_{k}^{q} P_{W_{k}^{1}}\left(D_{k}^{q}\right)^{\mathrm{T}}\right.} \\
& \left.+\sum_{q=1}^{3} \beta_{q}(1-\eta) \Phi S \underline{M}_{k}^{q} P_{\Lambda_{k}}\left(\underline{N}_{k}^{q}\right)^{\mathrm{T}}\right] \\
& \times\left[\sum_{q=1}^{3} \beta_{q} \widehat{C}_{k}^{q} P_{\xi_{k+1}}\left(\widehat{C}_{k}^{q}\right)^{\mathrm{T}}+\sum_{q=1}^{3} \beta_{q} D_{k}^{q} P_{W_{k}}\left(D_{k}^{q}\right)^{\mathrm{T}}\right. \\
& \left.+\sum_{q=1}^{3} \beta_{q}(1-\eta) \underline{N}_{k}^{q} P_{\Lambda_{k}}\left(\underline{N}_{k}^{q}\right)^{\mathrm{T}}\right]^{-1} .
\end{aligned}
$$

Therefore the covariance matrices of the estimation error for any (i.e., not necessarily optimal) $K_{k}$ can be computed by a set of recursive discrete-time Riccati-like equations. The corresponding optimal $G_{k}$ can be computed by

$$
G_{k}=\left[\begin{array}{ll}
K_{k}^{\mathrm{T}} & 0
\end{array}\right]^{\mathrm{T}}
$$

Let $\widehat{A}_{k}=I, B_{k}^{q}=0$, and $L_{k}=K_{k}$. We may obtain the relevant formula for measurement update as follows:

$$
\begin{aligned}
P_{e_{k+1 \mid k+1}}= & \sum_{q=1}^{3} \beta_{q}\left\{\left(\Phi-M_{k} \widehat{C}_{k}^{q}\right) P_{\xi_{k+1}}\left(\Phi-M_{k} \widehat{C}_{k}^{q}\right)^{\mathrm{T}}\right. \\
& \left.+L_{k} D_{k}^{q} P_{W_{k}}\left(D_{k}^{q}\right)^{\mathrm{T}} M_{k}^{\mathrm{T}}\right\} \\
& +(1-\eta) \sum_{q=1}^{3} \beta_{q} L_{k} \underline{N}_{k}^{q} P_{\Lambda_{k}}\left(\underline{N}_{k}^{q}\right)^{\mathrm{T}} \\
L_{k}= & {\left[\sum_{q=1}^{3} \beta_{q} \Phi P_{\xi_{k+1}}\left(\widehat{C}_{k}^{q}\right)^{\mathrm{T}}+(1-\eta) \Phi S \underline{M}_{k}^{q} P_{\Lambda_{k}}\left(\underline{N}_{k}^{q}\right)^{\mathrm{T}}\right] } \\
& \times\left[\sum_{q=1}^{3} \beta_{q} \widehat{C}_{k}^{q} P_{\xi_{k+1}}\left(\widehat{C}_{k}^{q}\right)^{\mathrm{T}}+\sum_{q=1}^{3} \beta_{q} D_{k}^{q} P_{W_{k}}\left(D_{k}^{q}\right)^{\mathrm{T}}\right. \\
& +\sum_{q=1}^{3} \beta_{q}(1-\eta) \underline{\left.N_{k}^{q} P_{\Lambda_{k}}\left(\underline{N_{k}^{q}}\right)^{\mathrm{T}}\right]^{-1} .}
\end{aligned}
$$

We may propose the following adaptive filtering scheme.

Consider the conceptual algorithm. 
Step 1. The required input parameters are

$$
F_{k}, H_{k}, \sigma_{w}^{2}, \sigma_{v}^{2}, \sigma_{\tau}^{2}, P_{0}, \rho_{q}(q=1,2,3), \quad E\left\{X_{0}\right\}=\mu_{0} .
$$

Step 2. Initialization is as follows:

$$
\begin{gathered}
\left\{A_{k}^{q}, B_{k}^{q}, C_{k}^{q}, D_{k}^{q}, M_{k}^{q}, N_{k}^{q} \mid q=1,2,3\right\}, \\
P_{W_{k}}=\operatorname{diag}\left\{\sigma_{w}^{2}, \sigma_{v}^{2}, \sigma_{v}^{2}\right\}, \\
P_{\Lambda_{k}}=\operatorname{diag}\left\{\sigma_{\tau}^{2}, \sigma_{\tau}^{2}\right\}, \\
P_{\xi_{0}}=\left[\begin{array}{ll}
P_{0} & P_{0} \\
P_{0} & P_{0}
\end{array}\right],
\end{gathered}
$$

where we have assumed $X_{-1}=X_{0}$ to derive the above initialization.

Perform the following equations:

$$
\begin{aligned}
\underline{X}_{0}=E\left\{\left[\begin{array}{ll}
X_{0}^{\mathrm{T}} & X_{0}^{\mathrm{T}}
\end{array}\right]^{\mathrm{T}}\right\}=\left[\begin{array}{ll}
\mu_{0}^{\mathrm{T}} & \mu_{0}^{\mathrm{T}}
\end{array}\right]^{\mathrm{T}}, \\
\widehat{C}_{0}=\sum_{q=1}^{3} \rho_{0}^{q} C_{0}^{q} S^{\mathrm{T}}, \\
K_{0}=\left(\sum_{q=1}^{3} \beta_{0}^{q} \Phi P_{\xi_{0}}\left(\widehat{C}_{0}^{q}\right)^{\mathrm{T}}\right) \\
\times\left(\sum _ { q = 1 } ^ { 3 } \beta _ { 0 } ^ { q } \left\{\widehat{C}_{0}^{q} P_{\xi_{0}}\left(\widehat{C}_{0}^{q}\right)^{\mathrm{T}}+D_{0}^{q} P_{W_{0}}\left(D_{0}^{q}\right)^{\mathrm{T}}\right.\right. \\
\left.\left.+(1-\eta) \underline{N}_{0}^{q} P_{\Lambda_{0}}\left(\underline{N}_{0}^{q}\right)^{\mathrm{T}}\right\}\right)^{-1}, \\
P_{e_{0 \mid 0}}=\sum_{q=1}^{3} \rho_{0}^{q}\left(I-K_{0} \widehat{C}_{0}^{q}\right) P_{\xi_{0}}\left(I-K_{0} \widehat{C}_{0}^{q}\right)^{\mathrm{T}} \\
\quad \times \sum_{q=1}^{3} \rho_{k}^{q} K_{0} D_{0}^{q} P_{W_{0}}\left(D_{0}^{q}\right)^{\mathrm{T}} K_{0}^{\mathrm{T}} \\
+\sum_{q=1}^{3} \rho_{0}^{q}(1-\eta) K_{0} \underline{N}_{0}^{q} P_{\Lambda_{0}}\left(\underline{N}_{0}^{q}\right)^{\mathrm{T}} K_{0}^{\mathrm{T}} .
\end{aligned}
$$

Step 3. Consider the state prediction

$$
\begin{gathered}
\widehat{X}_{k+1}=F \widehat{X}_{k \mid k}, \\
\widehat{X}_{k+1}=\left[\begin{array}{ll}
\widehat{X}_{k+1}^{\mathrm{T}}, & \widehat{X}_{k}^{\mathrm{T}}
\end{array}\right]^{\mathrm{T}}
\end{gathered}
$$

and the prediction covariance computation

$$
\begin{aligned}
P_{\xi_{k+1}}= & \widehat{A}_{k} P_{\xi_{k \mid k}} \widehat{A}_{k}^{\mathrm{T}} \\
& +\sum_{q=1}^{3} \beta_{k}^{q}\left\{S B_{k}^{q} P_{W_{k}}\left(B_{k}^{q}\right)^{\mathrm{T}} S^{\mathrm{T}}\right. \\
& \left.\quad+(1-\eta) S \underline{M}_{k}^{q} P_{\Lambda_{k}}\left(\underline{M}_{k}^{q}\right)^{\mathrm{T}} S^{\mathrm{T}}\right\} .
\end{aligned}
$$

Step 4. Innovation update is as follows.

$$
\text { If } d_{k}=0 \text {, then } K_{k}=0 \text {. Else if } d_{k}=1 \text {, then }
$$

$$
\begin{aligned}
K_{k}= & \left(\sum_{q=1}^{3} \beta_{k}^{q} \Phi P_{\xi_{k+1}}\left(\widehat{C}_{k}^{q}\right)^{\mathrm{T}}\right) \\
& \times\left(\sum _ { q = 1 } ^ { 3 } \beta _ { k } ^ { q } \left\{\widehat{C}_{k}^{q} P_{\xi_{k+1}}\left(\widehat{C}_{k}^{q}\right)^{\mathrm{T}}+D_{k}^{q} P_{W_{k}}\left(D_{k}^{q}\right)^{\mathrm{T}}\right.\right. \\
& \left.\left.+(1-\eta) \underline{N}_{k}^{q} P_{\Lambda_{k}}\left(\underline{N}_{k}^{q}\right)^{\mathrm{T}}\right\}\right)^{-1} .
\end{aligned}
$$

Step 5. Update $k=k+1$ :

$$
\widehat{X}_{k \mid k}=\widehat{X}_{k}+K_{k}\left(y_{k}-\widehat{C}_{k} \widehat{X}_{k}\right) .
$$

Considering error covariance matrix update, perform the following equations:

$$
\begin{gathered}
G_{k}=\left[\begin{array}{ll}
K_{k}^{\mathrm{T}} & 0
\end{array}\right]^{\mathrm{T}}, \\
P_{\xi_{k+1 \mid k+1}}=\sum_{q=1}^{3} \beta_{k}^{q}\left\{\left(I-G_{k} \widehat{C}_{k}^{q}\right) P_{\xi_{k}}\left(I-G_{k} \widehat{C}_{k}^{q}\right)^{\mathrm{T}}\right. \\
+G_{k} D_{k}^{q} P_{W_{k}}\left(D_{k}^{q}\right)^{\mathrm{T}} G_{k}^{\mathrm{T}} \\
\left.+(1-\eta) G_{k} \underline{N}_{k}^{q} P_{\Lambda_{k}}\left(\underline{N}_{k}^{q}\right)^{\mathrm{T}} G_{k}^{\mathrm{T}}\right\} .
\end{gathered}
$$

Remark 2. We consider that the model $\left\{A_{k}^{q}, B_{k}^{q}, C_{k}^{q}, D_{k}^{q}, M_{k}^{q}\right.$, $\left.N_{k}^{q} \mid q=1,2,3\right\}$ is unknown when the measurement arrives in an estimator. In this case, we need to know the probability $\rho_{q}(q=1,2,3)$ of each model. However, we can get an exact model $\left\{A_{k}^{q}, B_{k}^{q}, C_{k}^{q}, D_{k}^{q}, M_{k}^{q}, N_{k}^{q} \mid q=\right.$ $1,2,3\}$ when the measurement with time stamp arrives in an estimator, in which case, we may have an improved adaptive algorithm with time stamp.

\section{Simulation Results}

In this section, Monte Carlo simulations were conducted to verify the performance of the algorithms in three scenarios of target tracking: uniform rectilinear motion scenario, uniform circular motion scenario, and uniformly accelerated motion scenario. A total of 100 runs were executed in each case.

(A) Uniform Rectilinear Motion Scenario. Consider the system (1) with parameters

$$
\begin{gathered}
F_{k}=I_{3 \times 3} \otimes\left[\begin{array}{ll}
1 & T \\
0 & 1
\end{array}\right], \\
\sigma_{w}^{2}=I_{3 \times 3} \otimes\left[\begin{array}{cc}
\frac{T^{3}}{3} & \frac{T^{2}}{2} \\
\frac{T^{2}}{2} & T
\end{array}\right], \quad \sigma_{v}^{2}=25 \mathrm{~m}^{2}, \quad \sigma_{\tau}^{2}=25 \mathrm{~m}^{2},
\end{gathered}
$$

where $T$ is the sampling period, $\otimes$ is the Kronecker product, and $I_{3 \times 3}$ denotes the unity matrix of 3 orders.

Assume that the initial state of target is $X_{0}=[-8000,200$, $0,500,0,0,1000,0,0]^{\mathrm{T}}$, the sampling period is set to be 


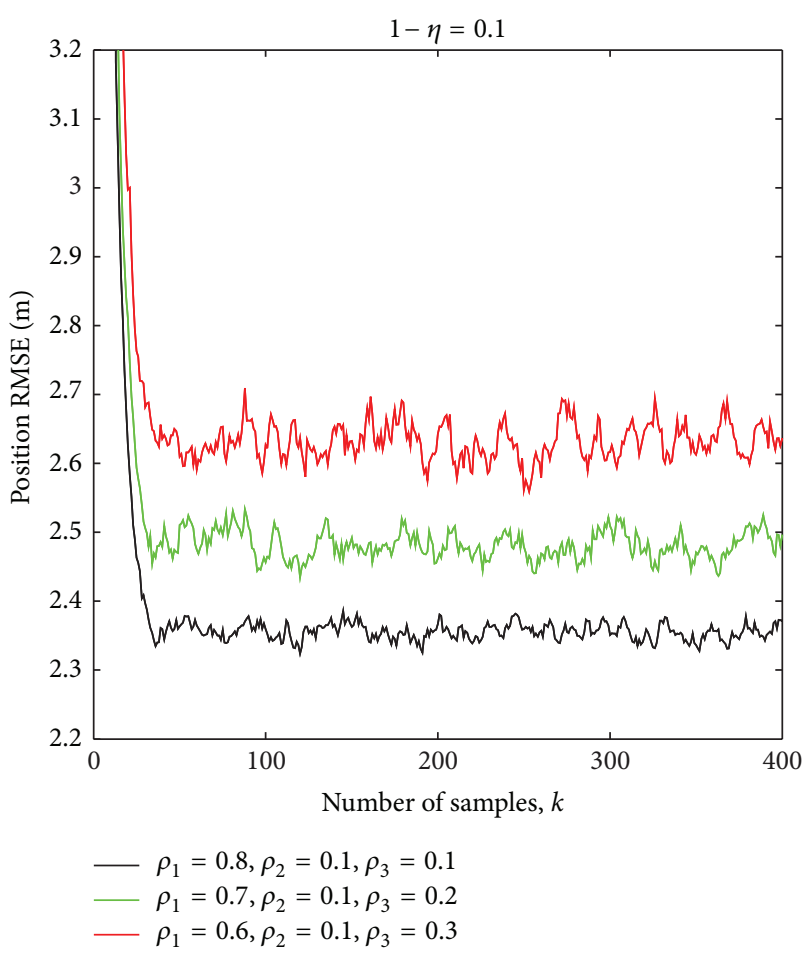

(a)

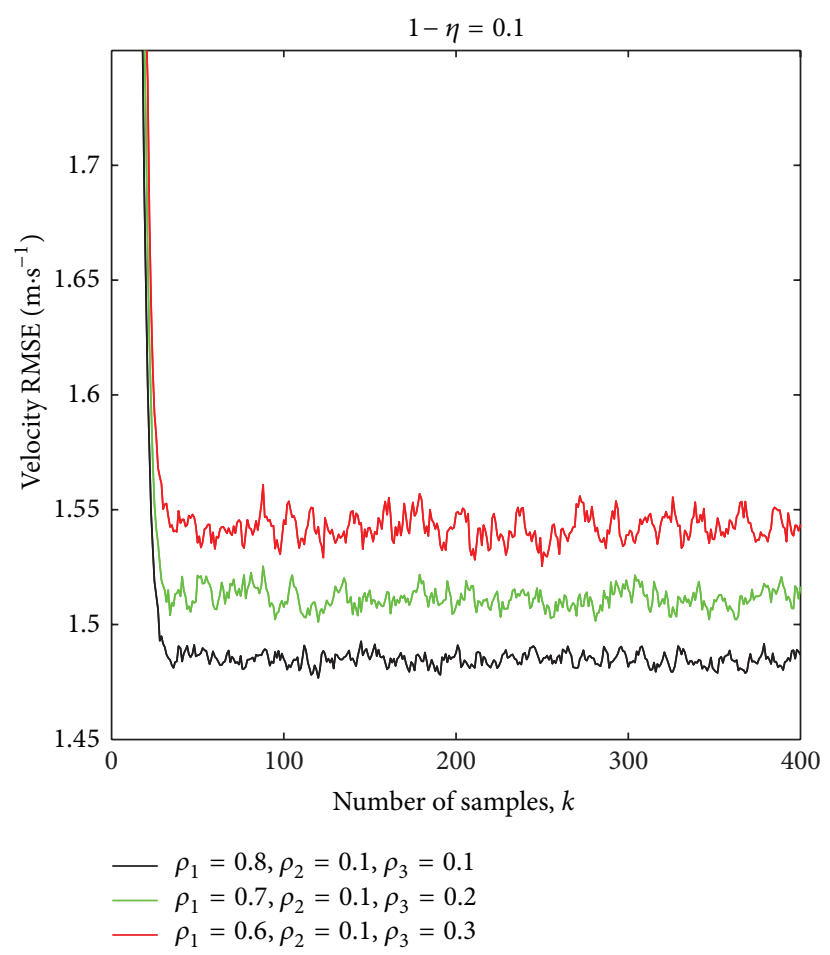

(b)

FIGURE 1: Comparison of the RMSEs for the proposed algorithm with different $\rho_{q}(q=1,2,3)$ : (a) position RMSE; (b) velocity RMSE.

$T=0.2 \mathrm{~s}$, the probability of stochastic-bias measurement is $1-\eta=0.1$. The simulation results are obtained by the proposed adaptive estimation algorithm in Section 4 . The root mean square error (RMSE) for the states of position and velocity with different $\rho_{q}(q=1,2,3)$ is given in Figures 14. Seen from Figures $1-4$, the proposed adaptive estimation algorithm is effective. Besides, it can be seen from Figure 1 that the tracking performance is better with higher probability when the delay probability is constant, where the black curves denote position and velocity RMSEs, respectively, with detection probability $\rho_{1}=0.8$, delay probability $\rho_{2}=0.1$, and the probability of missing probability $\rho_{3}=0.1$, the green curve with $\rho_{1}=0.7, \rho_{2}=0.1$, and $\rho_{3}=0.2$, and the red curve with $\rho_{1}=0.6, \rho_{2}=0.1$, and $\rho_{3}=0.3$. Figure 2 gives comparison curves of position RMSE and velocity RMSE, respectively, where the black curves denote position RMSE with $\rho_{1}=0.8, \rho_{2}=0.1$, and $\rho_{3}=0.1$, the green curve with $\rho_{1}=0.8, \rho_{2}=0.05$, and $\rho_{3}=0.15$, and the red curve with $\rho_{1}=0.8, \rho_{2}=0.15$, and $\rho_{3}=0.05$. From Figure 3 , it is obvious that the estimation performance is better with lower missing measurement when the detection probability is constant. From Figure 4, we can see that the estimation algorithm has worse performance with a higher delay probability when the probability of missing measurement is constant.

Next, we consider the tracking performances with stochastic-bias probability $1-\eta$. Figure 4 gives comparison curves of position RMSE and velocity RMSE with $\rho_{1}=0.8$, $\rho_{2}=0.1$, and $\rho_{3}=0.1$, respectively, where the black curves denote position RMSE and velocity RMSE with $1-\eta=0.1$, the green curve with $1-\eta=0.5$, and the red curve with $1-\eta=0.8$. From Figure 4 , we can obtain that the estimation performance is better with lower probability of stochasticbias measurement.

To further demonstrate the effectiveness of our proposed algorithm, simulation scenarios of uniform rectilinear motion and uniform accelerated motion are given in the following.

(B) Uniform Rectilinear Motion Scenario. Consider the system (1) with parameters.

$$
\begin{gathered}
F_{k}=I_{3 \times 3} \otimes\left[\begin{array}{ccc}
1 & \frac{\sin (\omega T)}{\omega} & \frac{[1-\cos (\omega T)]}{\omega^{2}} \\
0 & \cos (\omega T) & \frac{\sin (\omega T)}{\omega} \\
0 & -\omega \sin (\omega T) & \cos (\omega T)
\end{array}\right], \\
\sigma_{w}^{2}=I_{3 \times 3} \otimes S, \quad \sigma_{v}^{2}=25 \mathrm{~m}^{2}, \\
S=\left[\begin{array}{lll}
A_{11} & A_{12} & A_{13} \\
A_{21} & A_{22} & A_{23} \\
A_{31} & A_{32} & A_{33}
\end{array}\right], \\
A_{11}=6 \omega T-8 \sin (\omega T)+\frac{\sin (2 \omega T)}{4 \omega^{5}}, \\
A_{12}=\frac{[2 \sin (\omega T / 2)]^{4}}{\omega^{4}},
\end{gathered}
$$




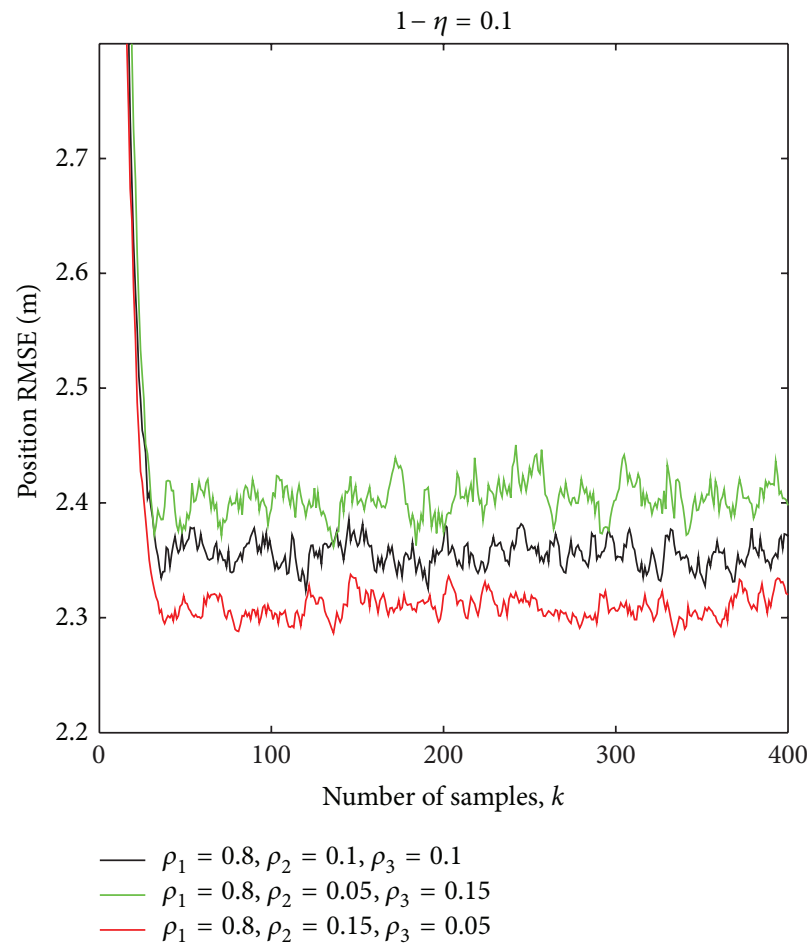

(a)

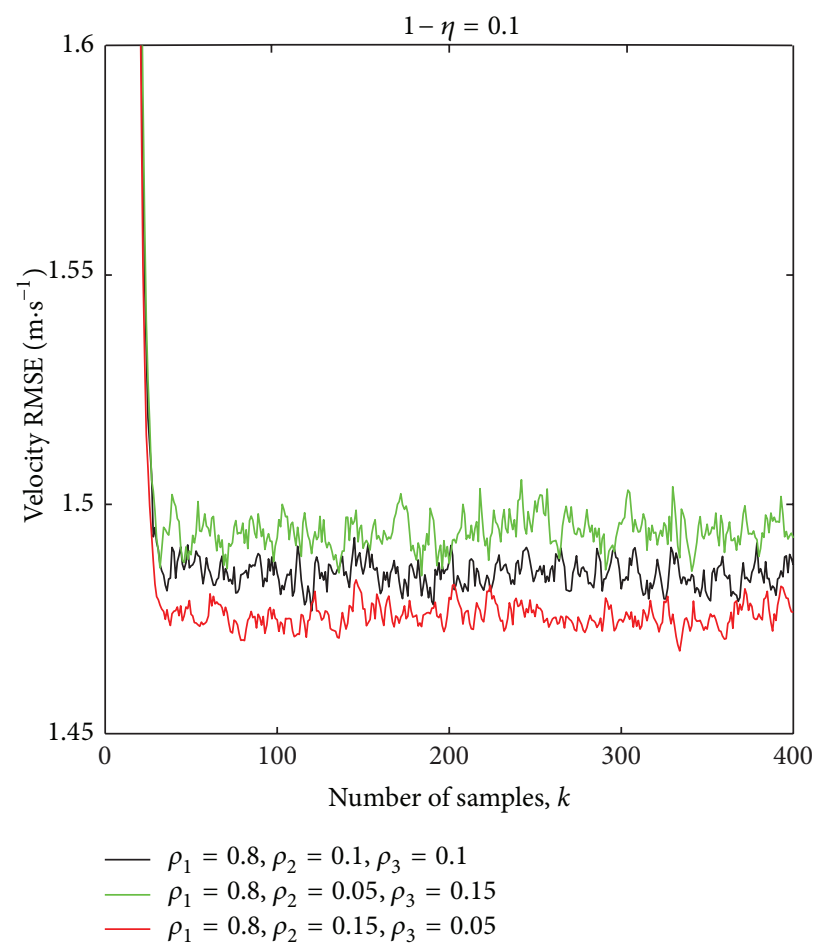

(b)

FIgURE 2: Comparison of the RMSEs for the proposed algorithm with different $\rho_{q}(q=1,2,3)$ : (a) position RMSE; (b) velocity RMSE.

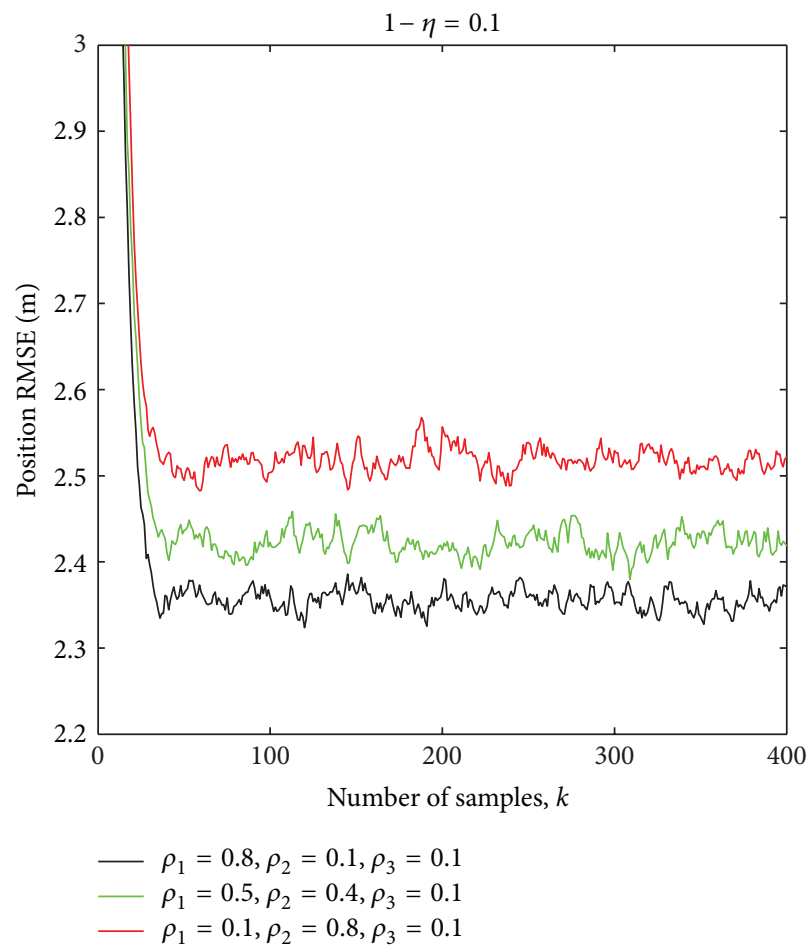

(a)

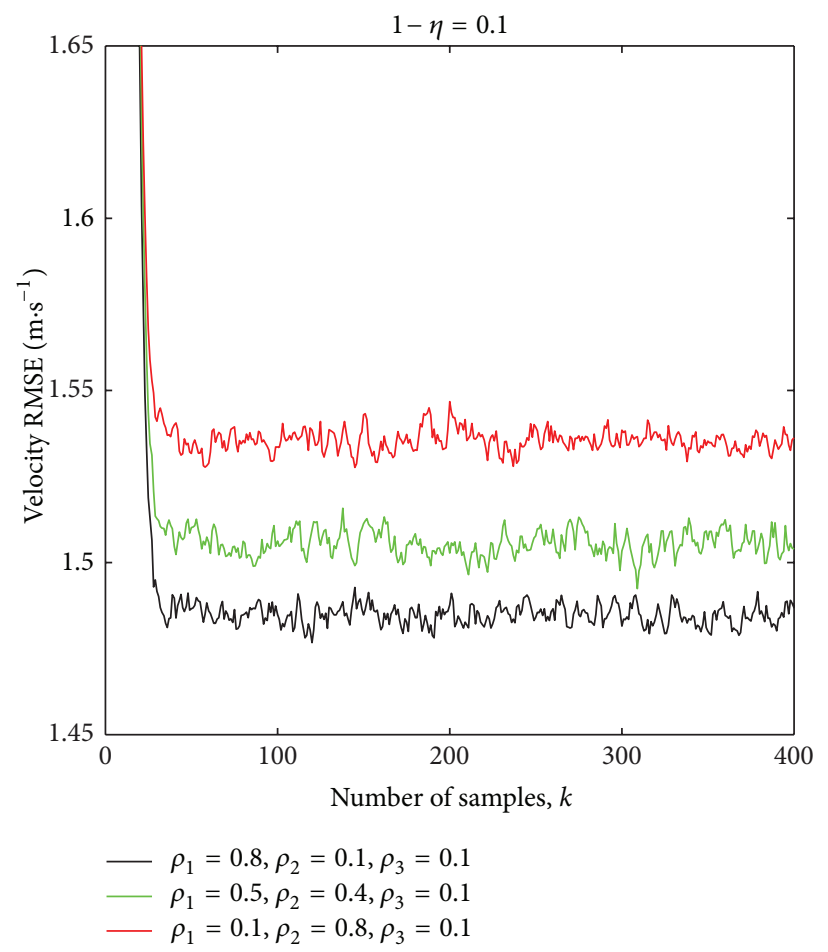

(b)

FIgURE 3: Comparison of the RMSEs for the proposed algorithm with different $\rho_{q}(q=1,2,3)$ : (a) position RMSE; (b) velocity RMSE. 

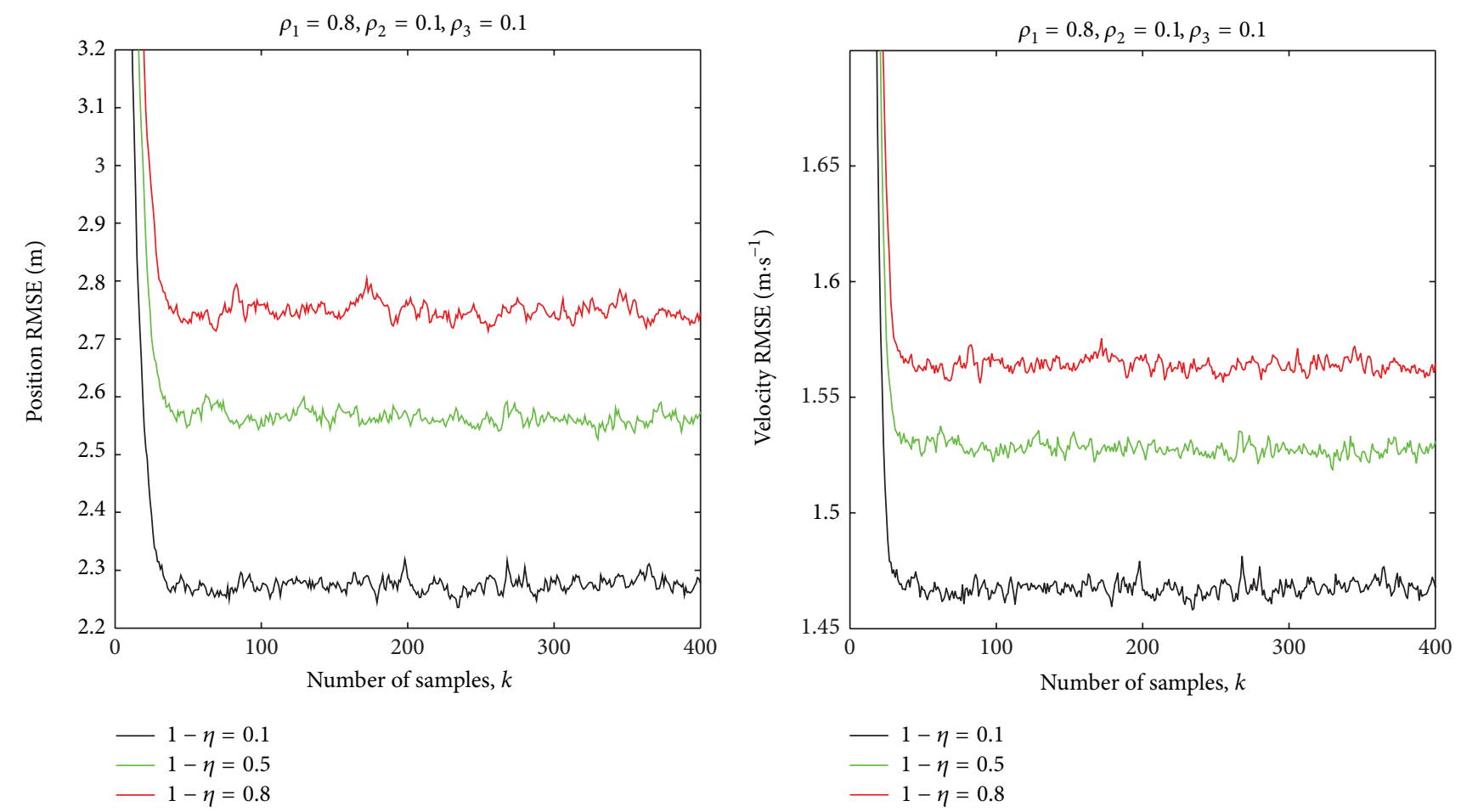

(a)

(b)

FIGURE 4: Comparison of the RMSEs for the proposed algorithm with different $\rho_{q}(q=1,2,3)$ : (a) position RMSE; (b) velocity RMSE.

$$
\begin{gathered}
A_{13}=\frac{[-2 \omega T+4 \sin (\omega T)-\sin (2 \omega T)]}{4 \omega^{3}}, \\
A_{21}=\frac{2[\sin (\omega T / 2)]^{4}}{\omega^{4}}, \\
A_{22}=\frac{[2 \omega T-\sin (2 \omega T)]}{4 \omega^{3}}, \\
A_{31}=\frac{[-2 \omega T+4 \sin (\omega T)-\sin (2 \omega T)]}{4 \omega^{3}}, \\
A_{32}=\frac{[\sin (\omega T)]^{2}}{2 \omega^{2}}, \\
A_{33}=\frac{[2 \omega T+\sin (2 \omega T)]}{4 \omega},
\end{gathered}
$$

where $T$ is the sampling period, $\otimes$ is the Kronecker product, and $I_{3 \times 3}$ denotes the unity matrix of 3 orders.

Assume that the initial state of target is $X_{0}=[-5000$, $200,-1,500,0,0,1000,0,0]^{\mathrm{T}}$, the sampling period is set to be $T=0.2 \mathrm{~s}$, the angular velocity is set to be $\omega=$ $0.05 \mathrm{rad} / \mathrm{s}$, and the probability of stochastic-bias measurement is $1-\eta=0.1$. The simulation results can be obtained as in Figures 5, 6, 7, and 8 in this scenario.
(C) Uniform Accelerated Motion Scenario. Consider the system (1) with parameters

$$
\begin{gathered}
F_{k}=I_{3 \times 3} \otimes\left[\begin{array}{ccc}
1 & T & \frac{T^{2}}{2} \\
0 & 1 & T \\
0 & 0 & 1
\end{array}\right], \\
\sigma_{w}^{2}=I_{3 \times 3} \otimes\left[\begin{array}{ccc}
\frac{T^{5}}{20} & \frac{T^{4}}{8} & \frac{T^{3}}{6} \\
\frac{T^{4}}{8} & \frac{T^{3}}{3} & \frac{T^{2}}{2} \\
\frac{T^{2}}{2} & \frac{T^{2}}{2} & T
\end{array}\right], \\
\sigma_{v}^{2}=25 \mathrm{~m}^{2}, \quad \sigma_{\tau}^{2}=25 \mathrm{~m}^{2},
\end{gathered}
$$

where $T$ is the sampling period, $\otimes$ is the Kronecker product, and $I_{3 \times 3}$ denotes the unity matrix of 3 orders. Assume that the initial state of target is $X_{0}=[-5000,100,-5,500,0,0$, $1000,0,0]^{\mathrm{T}}$, the sampling period is set to be $T=0.2 \mathrm{~s}$, and the probability of stochastic-bias measurement is $1-\eta=0.1$. The simulation results can be obtained as in Figures 9, 10, 11, and 12 in this scenario.

From Figures 5-8 in scenario B and Figures 9-12 in scenario $C$, it is clear that the proposed algorithm is effective. On the other hand, the estimation performance can be influenced 


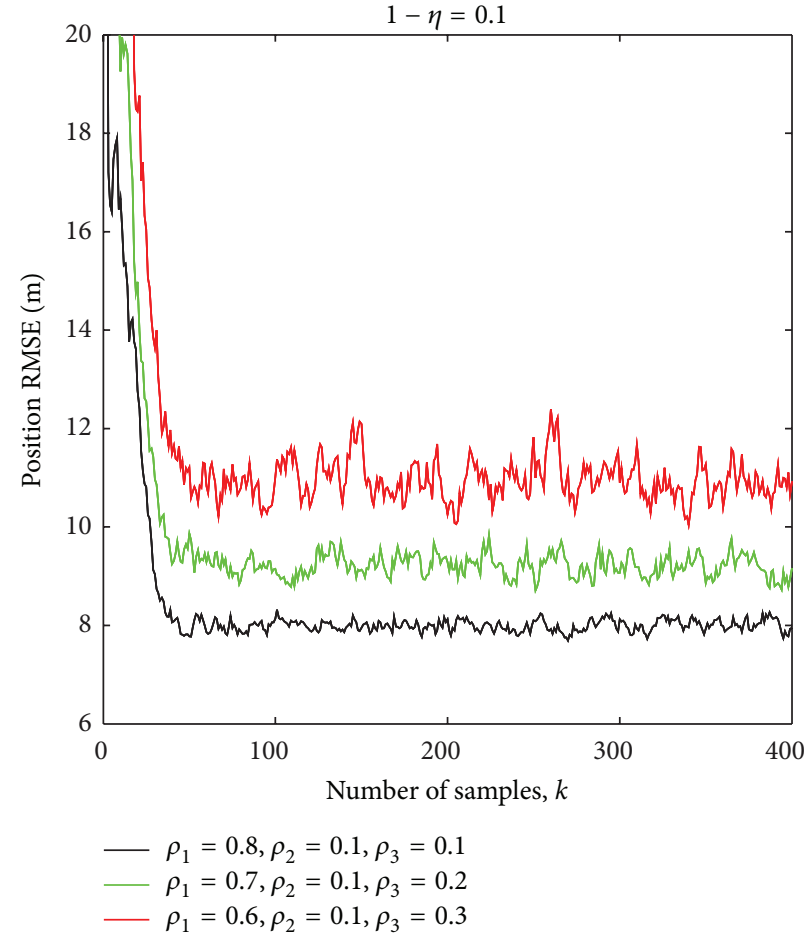

(a)

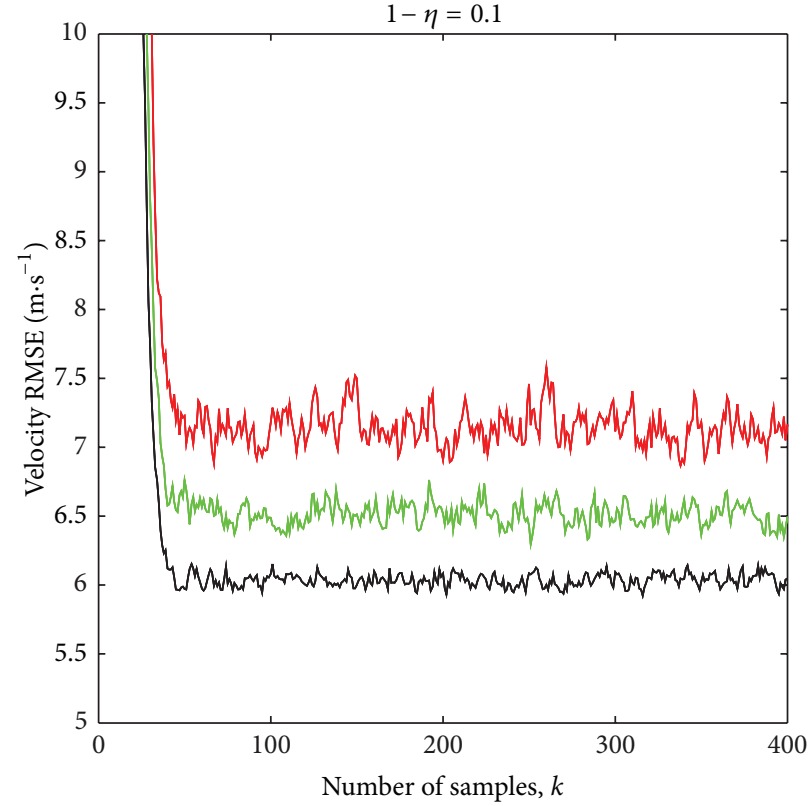

$\rho_{1}=0.8, \rho_{2}=0.1, \rho_{3}=0.1$

$\rho_{1}=0.7, \rho_{2}=0.1, \rho_{3}=0.2$

$\rho_{1}=0.6, \rho_{2}=0.1, \rho_{3}=0.3$

(b)

FIGURE 5: Comparison of the RMSEs for the proposed algorithm with different $\rho_{q}(q=1,2,3)$ : (a) position RMSE; (b) velocity RMSE.

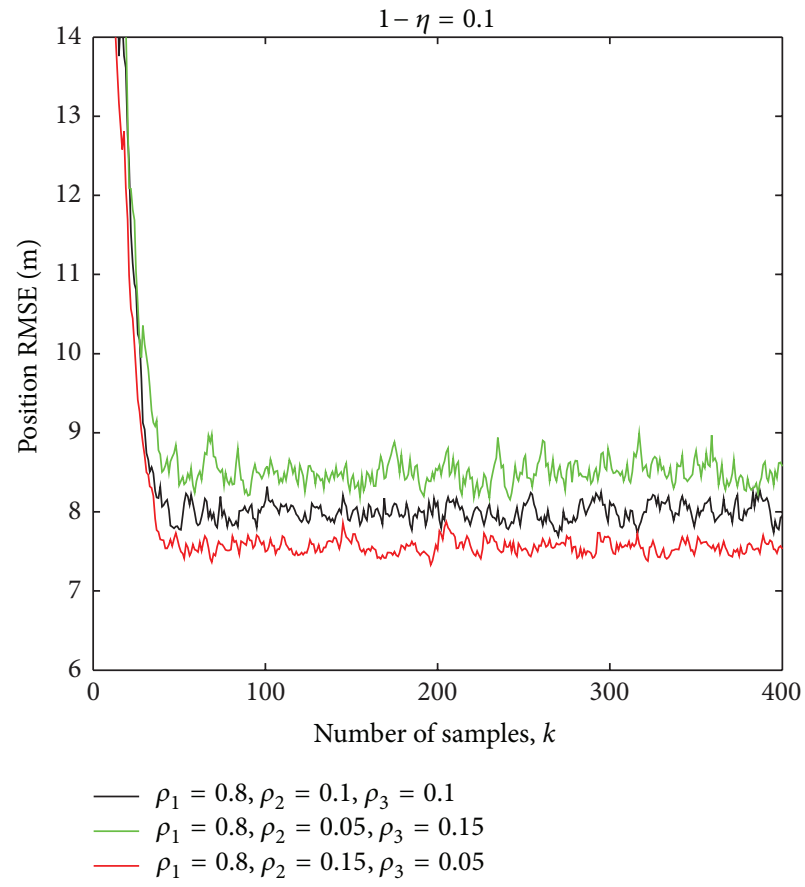

(a)

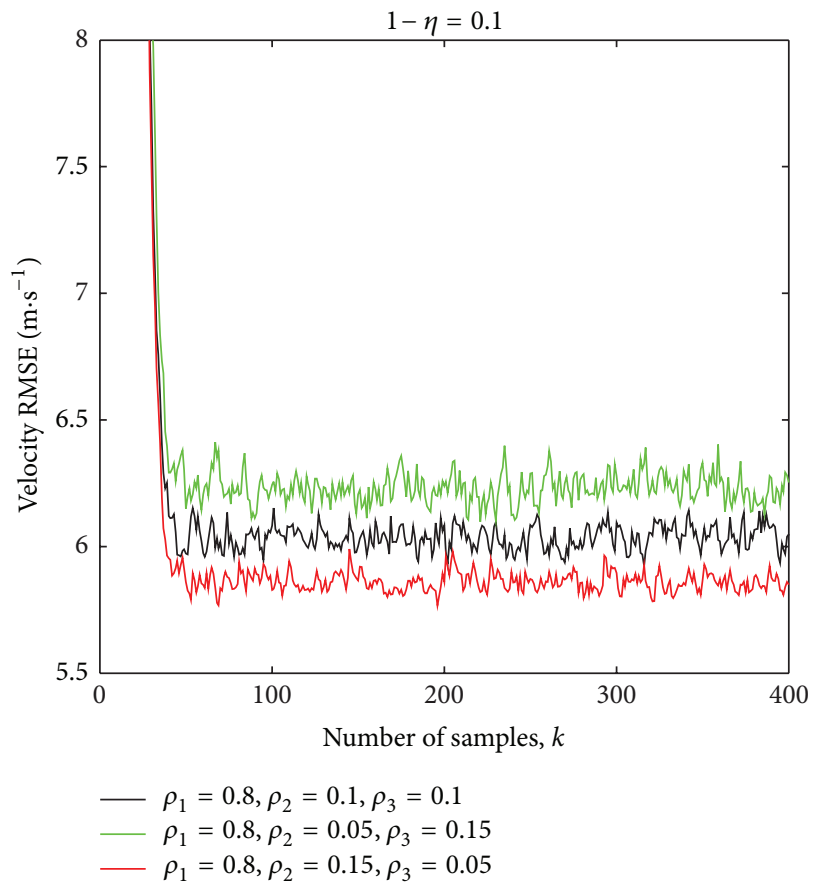

(b)

FIgURE 6: Comparison of the RMSEs for the proposed algorithm with different $\rho_{q}(q=1,2,3)$ : (a) position RMSE; (b) velocity RMSE. 


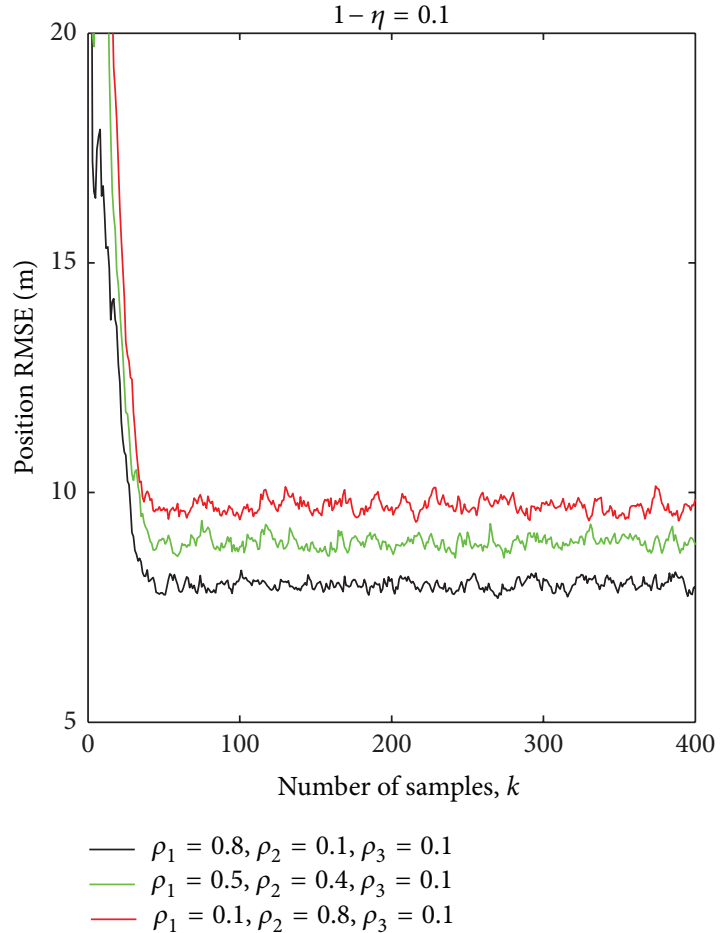

(a)

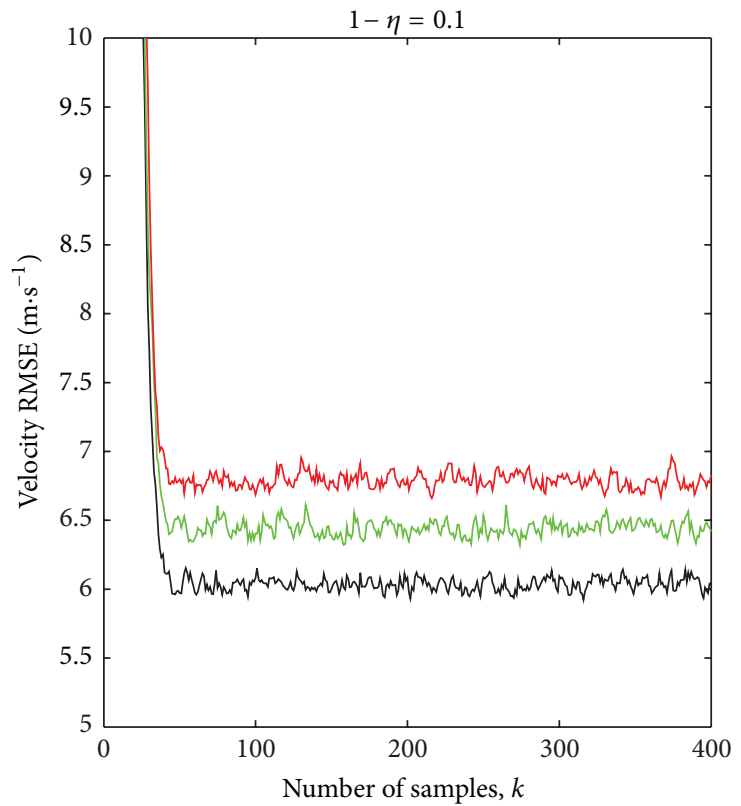

$\begin{aligned} \rho_{1} & =0.8, \rho_{2}=0.1, \rho_{3}=0.1 \\ \rho_{1} & =0.5, \rho_{2}=0.4, \rho_{3}=0.1 \\ \rho_{1} & =0.1, \rho_{2}=0.8, \rho_{3}=0.1\end{aligned}$

(b)

FIGURE 7: Comparison of the RMSEs for the proposed algorithm with different $\rho_{q}(q=1,2,3)$ : (a) position RMSE; (b) velocity RMSE.

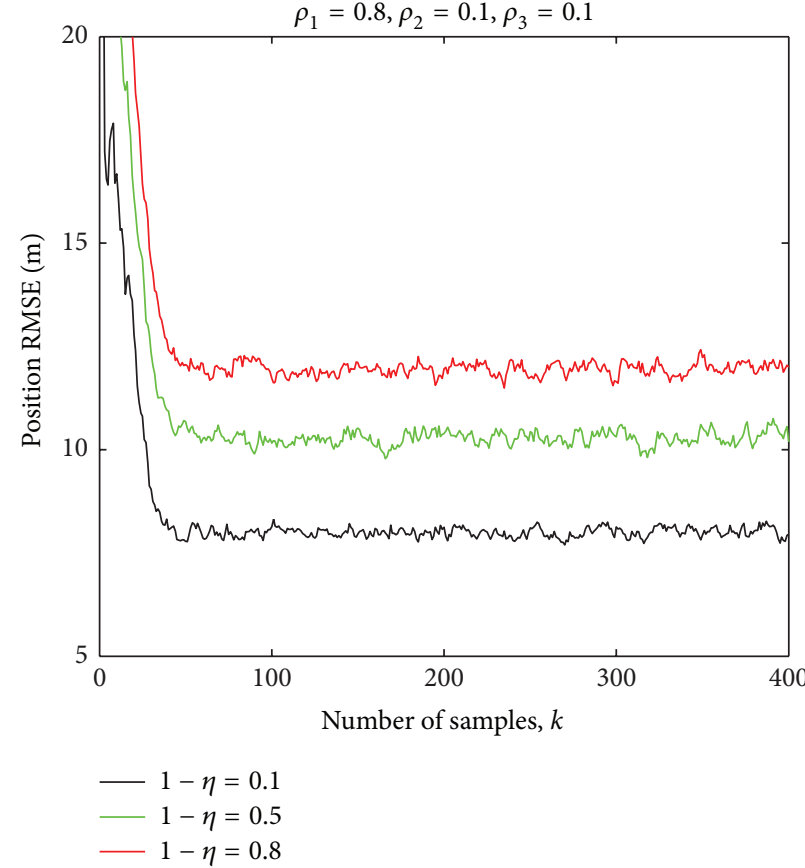

(a)

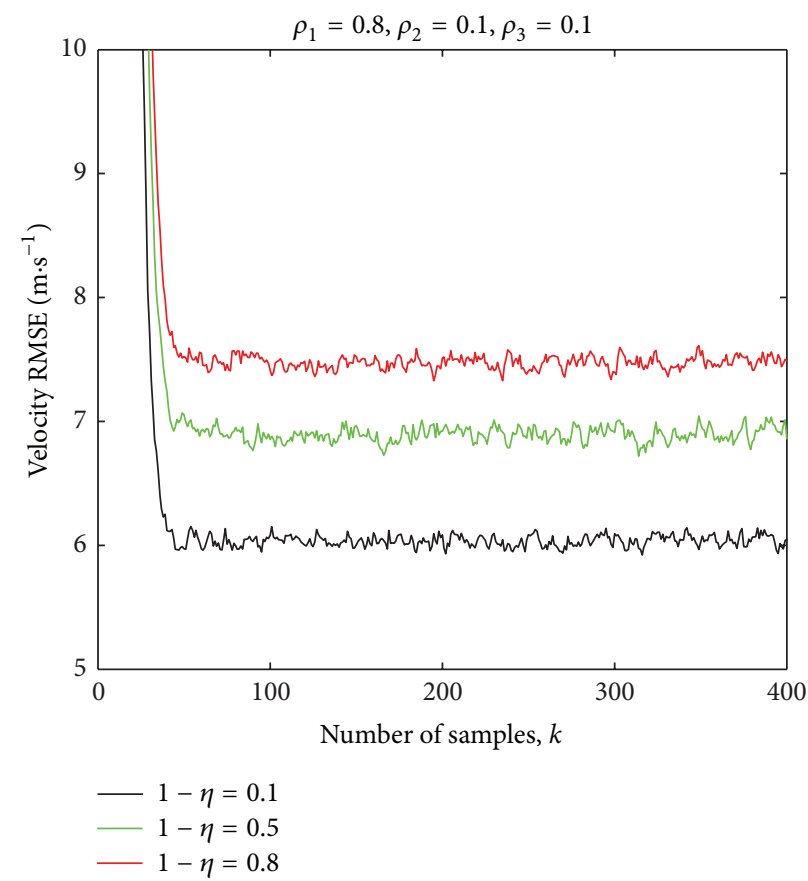

(b)

FIGURE 8: Comparison of the RMSEs for the proposed algorithm with different $\rho_{q}(q=1,2,3)$ : (a) position RMSE; (b) velocity RMSE. 

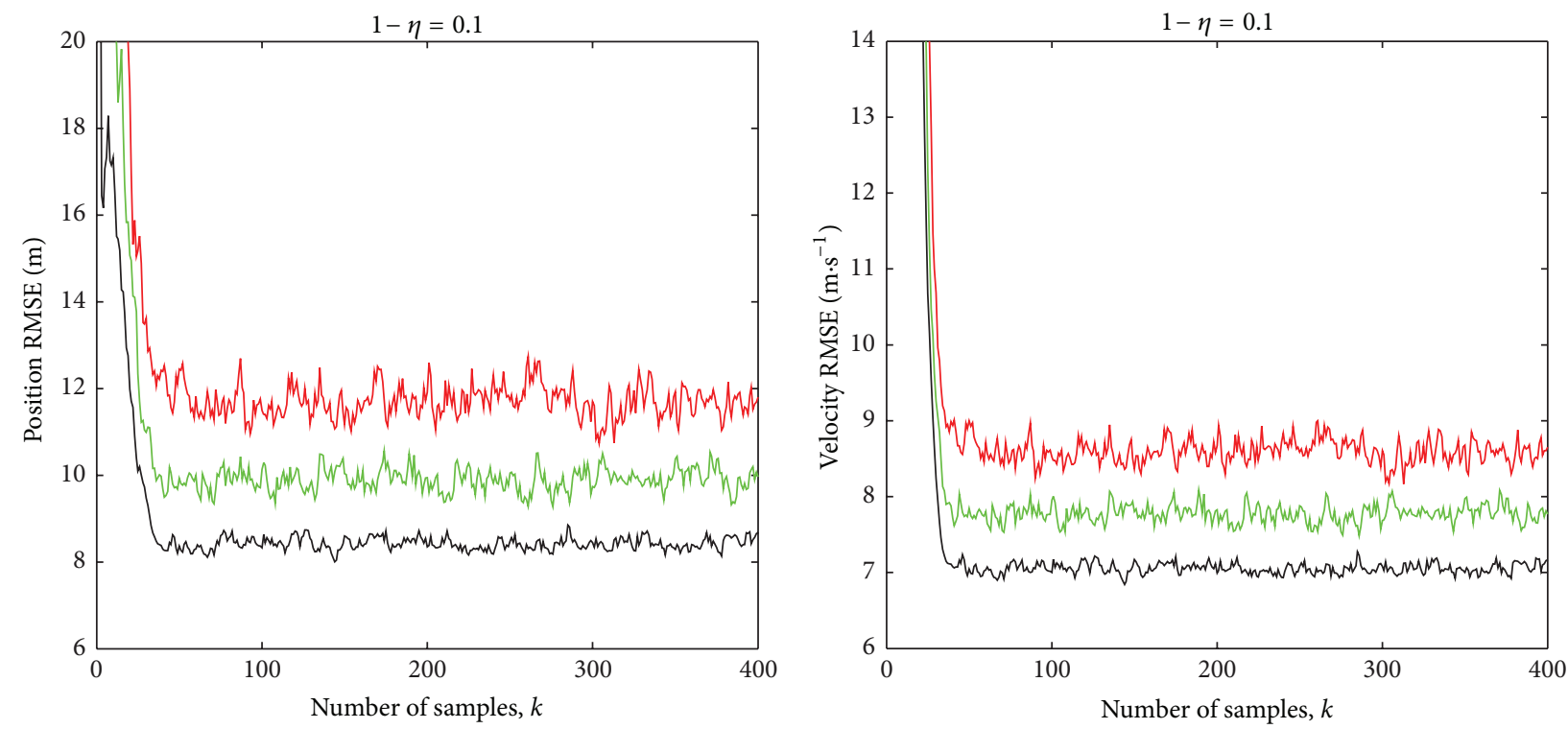

$$
\begin{aligned}
-\rho_{1} & =0.8, \rho_{2}=0.1, \rho_{3}=0.1 \\
-\rho_{1} & =0.7, \rho_{2}=0.1, \rho_{3}=0.2 \\
-\rho_{1} & =0.6, \rho_{2}=0.1, \rho_{3}=0.3
\end{aligned}
$$

(a)

$$
\begin{aligned}
-\rho_{1} & =0.8, \rho_{2}=0.1, \rho_{3}=0.1 \\
-\rho_{1} & =0.7, \rho_{2}=0.1, \rho_{3}=0.2 \\
-\rho_{1} & =0.6, \rho_{2}=0.1, \rho_{3}=0.3
\end{aligned}
$$

(b)

FIGURE 9: Comparison of the RMSEs for the proposed algorithm with different $\rho_{q}(q=1,2,3)$ : (a) position RMSE; (b) velocity RMSE.
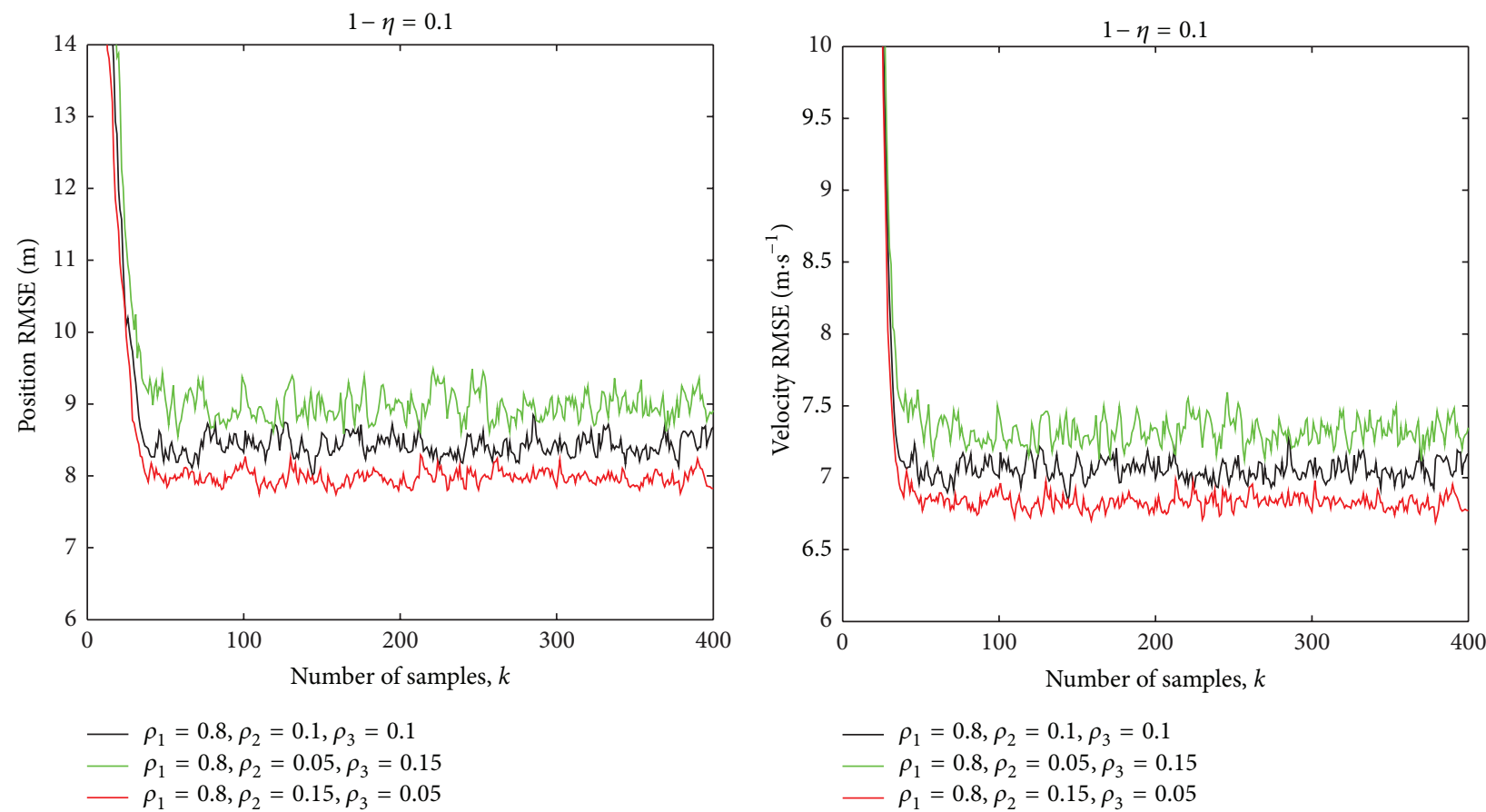

(a)

(b)

FIGURE 10: Comparison of the RMSEs for the proposed algorithm with different $\rho_{q}(q=1,2,3)$ : (a) position RMSE; (b) velocity RMSE. 


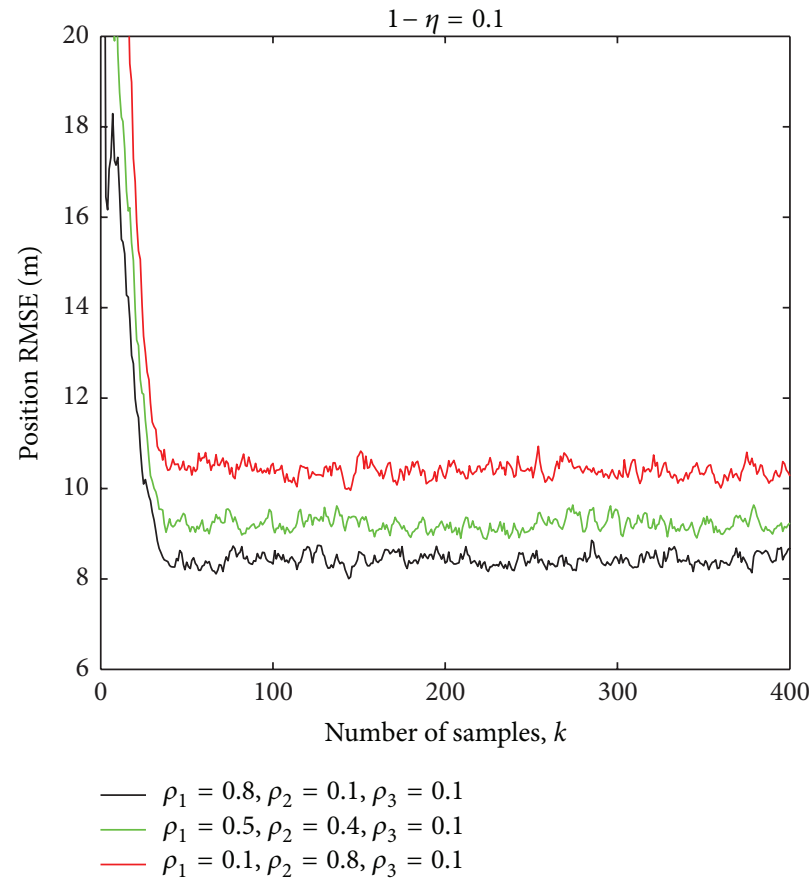

(a)

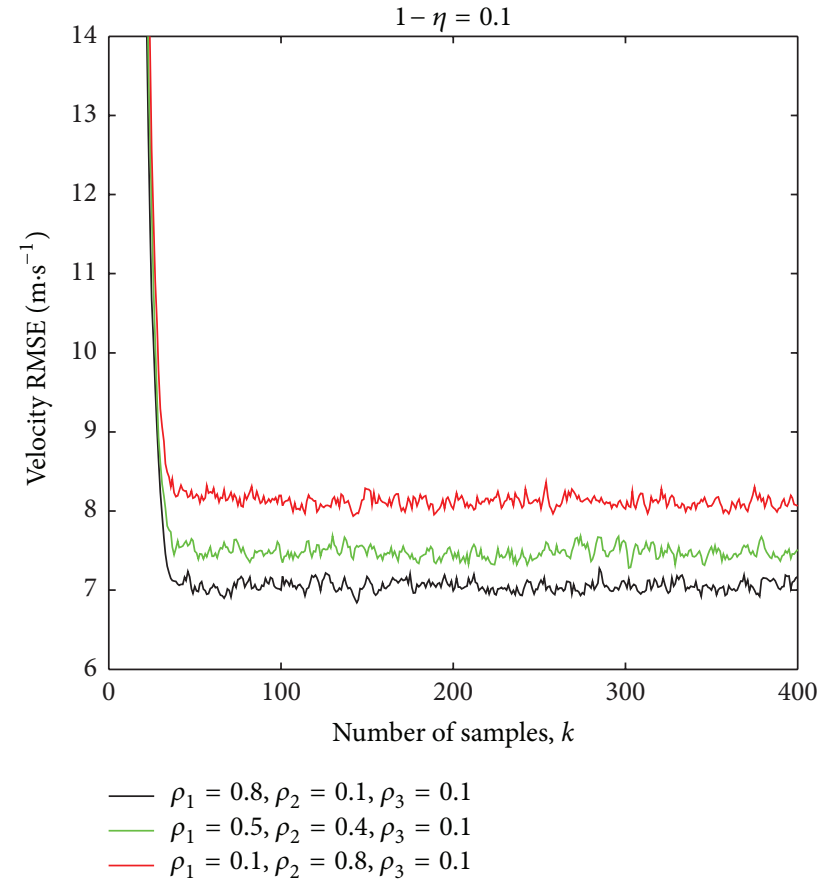

(b)

FIGURE 11: Comparison of the RMSEs for the proposed algorithm with different $\rho_{q}(q=1,2,3)$ : (a) position RMSE; (b) velocity RMSE.

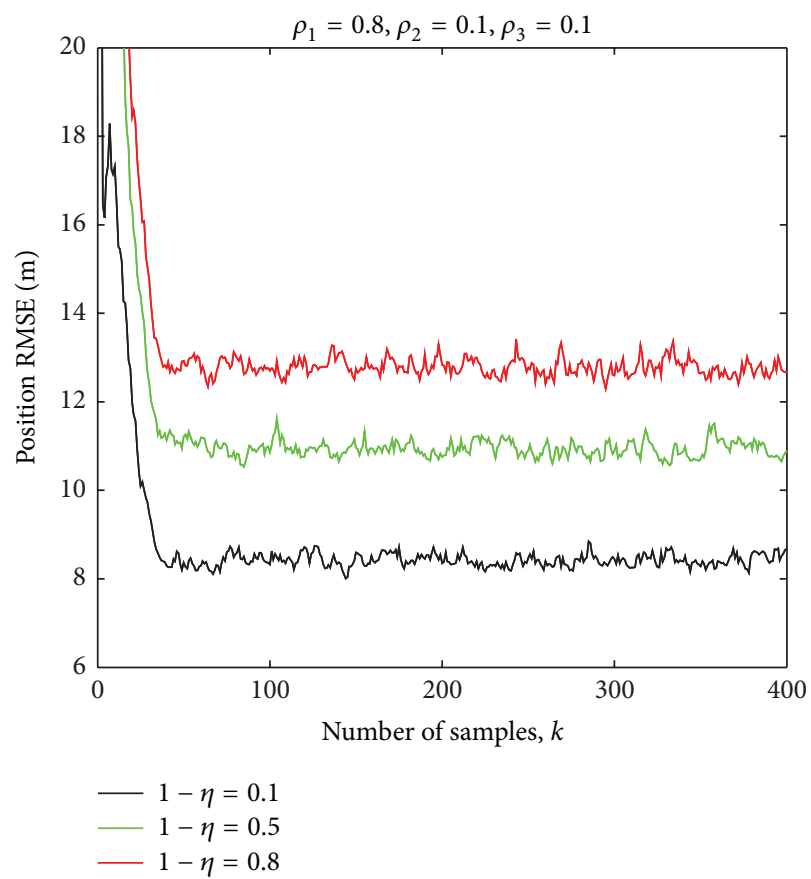

(a)

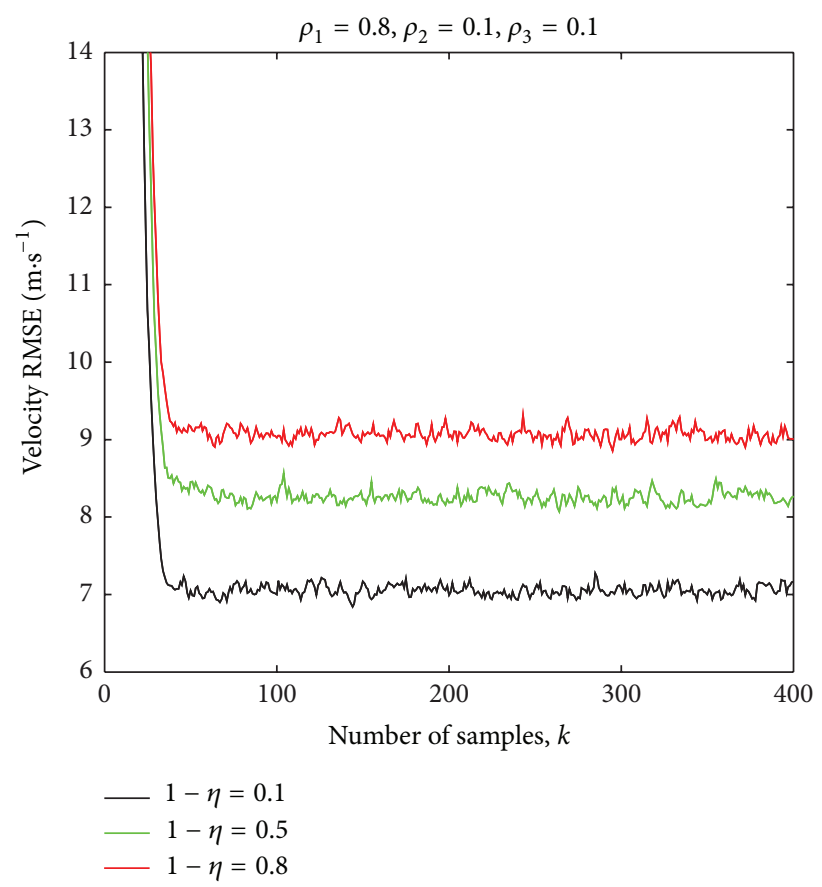

(b)

FIGURE 12: Comparison of the RMSEs for the proposed algorithm with different $\rho_{q}(q=1,2,3)$ : (a) position RMSE; (b) velocity RMSE.

by detection probability, one-step delay probability, missing probability, and stochastic-bias probability. Moreover, we have the corresponding results in scenarios $\mathrm{B}$ and $\mathrm{C}$ as well as in scenario A.

\section{Conclusions}

This paper presented an adaptive estimation algorithm in target tracking mixed with one-step delayed measurements, 
stochastic-bias measurements, and missing measurements. The contributions of this paper are as follows. First, the stochastic-bias measurement was incorporated into the proposed adaptive estimation algorithm. Second, the adaptive estimation algorithm mixed with one-step delayed measurements, stochastic-bias measurements, and missing measurements was implemented by augmenting the state vector. Examples and simulations are given to demonstrate the performances of the designed estimator. Through Monte Carlo simulations, the proposed algorithm was shown to be effective. Besides that, all the probabilities of missing measurements, stochastic-bias measurements, and one-step randomly delayed measurements have an influence on the estimation performance. Moreover, the estimation performance can be influenced by stochastic-bias probability. In this paper, we have only considered one-step sensor delays; furthermore, we can obtain the corresponding approach with multiple-step sensor delays by enlarging the state vectors.

\section{Acknowledgments}

This work was supported by the National Natural Science Foundations of China (nos. 61104186, and 61273076) and the Natural Science Foundation of Jiangsu Province (no. BK2012801) and sponsored by Research and Innovation Project for College Graduates of Jiangsu Province (2011) (no. CXLX11-0261).

\section{References}

[1] A. Farina, B. Ristic, and L. Timmoneri, "Cramér-Rao bound for nonlinear filtering with $P_{d}<1$ and its application to target tracking," IEEE Transactions on Signal Processing, vol. 50, no. 8, pp. 1916-1924, 2002.

[2] S. Nakamori, R. Caballero-Águila, A. Hermoso-Carazo, and J. Linares-Pérez, "New design of estimators using covariance information with uncertain observations in linear discrete-time systems," Applied Mathematics and Computation, vol. 135, no. 23, pp. 429-441, 2003.

[3] B. Sinopoli, L. Schenato, M. Franceschetti, K. Poolla, M. I. Jordan, and S. S. Sastry, "Kalman filtering with intermittent observations," IEEE Transactions on Automatic Control, vol. 49, no. 9, pp. 1453-1464, 2004.

[4] Z. Wang, D. W. C. Ho, and X. Liu, "Variance-constrained control for uncertain stochastic systems with missing measurements," IEEE Transactions on Systems, Man, and Cybernetics A, vol. 35, no. 5, pp. 746-753, 2005.

[5] Y. Yang, G. Feng, and J. Ren, "A combined backsteppingand small-gain approach to robust adaptive fuzzy control for strictfeedback nonlinear systems," IEEE Transactions on Systems, Man and Cybernetics A, vol. 34, no. 3, pp. 406-420, 2004.

[6] S. Nakamori, R. Caballero-Águila, A. Hermoso-Carazo, and J. Linares-Pérez, "Recursive estimators of signals from measurements with stochastic delays using covariance information," Applied Mathematics and Computation, vol. 162, no. 1, pp. 65-79, 2005.

[7] H. Dong, Z. Wang, D. W. C. Ho, and H. Gao, "Varianceconstrained $H_{\infty}$ filtering for a class of nonlinear time-varying systems with multiple missing measurements: the finitehorizon case," IEEE Transactions on Signal Processing, vol. 58, no. 5, pp. 2534-2543, 2010.

[8] S. Kluge, K. Reif, and M. Brokate, "Stochastic stability of the extended Kalman filter with intermittent observations," IEEE Transactions on Automatic Control, vol. 55, no. 2, pp. 514-518, 2010.

[9] Y. Mo and B. Sinopoli, "A characterization of the critical value for Kalman filtering with intermittent observations," in Proceedings of the 47th IEEE Conference on Decision and Control (CDC '08), pp. 2692-2697, Cancun, Mexico, December 2008.

[10] M. Moayedi, Y. K. Foo, and Y. C. Soh, "Adaptive Kalman filtering in networked systems with random sensor delays, multiple packet dropouts and missing measurements," IEEE Transactions on Signal Processing, vol. 58, no. 3, pp. 1577-1588, 2010.

[11] Y. Che, H. Shu, and X. Kan, "Estimation for stochastic nonlinear systems with randomly distributed time-varying delays and missing measurements," Mathematical Problems in Engineering, vol. 2012, Article ID 890512, 15 pages, 2012.

[12] L. Jinling, W. Zidong, and L. Xiaohui, "Robust state estimation for two-dimensional stochastic time-delay systems with missing measurements and sensor saturation," Multidimensional Systems and Signal Processing, 2012.

[13] L. Li and Y. Xia, "Stochastic stability of the unscented Kalman filter with intermittent observations," Automatica, vol. 48, no. 5, pp. 978-981, 2012.

[14] H. Dong, Z. Wang, and H. Gao, "Distributed filtering for a class of time-varying systems over sensor networks with quantization errors and successive packet dropouts," IEEE Transactions on Signal Processing, vol. 60, no. 6, pp. 3164-3173, 2012.

[15] R. Niu, P. Willett, and Y. Bar-Shalom, "Matrix CRLB scaling due to measurements of uncertain origin," IEEE Transactions on Signal Processing, vol. 49, no. 7, pp. 1325-1335, 2001.

[16] A. Farina, L. Timmoneri, S. Immediata, and B. Ristic, "CRLB with $P_{d}<1$ fused tracks," in Proceedings of the 8th International Conference on Information Fusion (FUSION '05), pp. 191-196, July 2005.

[17] Y. Boers and H. Driessen, "Results on the modified Riccati equation: target tracking applications," IEEE Transactions on Aerospace and Electronic Systems, vol. 42, no. 1, pp. 379-384, 2006.

[18] Y. Boers and H. Driessen, "Modified Riccati equation and its application to target tracking," IEE Proceedings: Radar, Sonar and Navigation, vol. 153, no. 1, pp. 7-12, 2006.

[19] H. Zhang, Q. Chen, H. Yan, and J. Liu, "Robust $H_{\infty}$ filtering for switched stochastic system with missing measurements," IEEE Transactions on Signal Processing, vol. 57, no. 9, pp. 3466-3474, 2009.

[20] A. Censi, "Kalman filtering with intermittent observations: convergence for semi-Markov chains and an intrinsic performance measure," IEEE Transactions on Automatic Control, vol. 56, no. 2, pp. 376-381, 2011.

[21] H. Dong, Z. Wang, and H. Gao, "Distributed $H_{\infty}$ filtering for a class of Markovian jump nonlinear time-delay systems over lossy sensor networks," IEEE Transactions on Industrial Electronics, vol. 60, no. 10, pp. 4665-4672, 2013.

[22] A. Ray, L. W. Liou, and J. H. Shen, "State estimation using randomly delayed measurements," Journal of Dynamic Systems, Measurement and Control, vol. 115, no. 1, pp. 19-26, 1993.

[23] J. Nilsson, B. Bernhardsson, and B. Wittenmark, "Stochastic analysis and control of real-time systems with random time delays," Automatica, vol. 34, no. 1, pp. 57-64, 1998. 
[24] Z. Wang, F. Yang, D. W. C. Ho, and X. Liu, "Robust $H_{\infty}$ filtering for stochastic time-delay systems with missing measurements," IEEE Transactions on Signal Processing, vol. 54, no. 7, pp. 25792587, 2006.

[25] F. Yang, Z. Wang, G. Feng, and X. Liu, "Robust filtering with randomly varying sensor delay: the finite-horizon case," IEEE Transactions on Circuits and Systems I, vol. 56, no. 3, pp. 664672, 2009.

[26] X. Wang, Q. Pan, Y. Liang, and F. Yang, "Gaussian smoothers for nonlinear systems with one-step randomly delayed measurements," IEEE Transactions on Automatic Control, vol. 58, no. 7, pp. 1828-1835, 2013.

[27] S. Elmadssia, K. Saadaoui, and M. Benrejeb, "New delaydependent stability conditions for linear systems with delay," Systems Science and Control Engineering, vol. 1, no. 1, pp. 2-11, 2013.

[28] A. T. Alouani, P. Xia, T. R. Rice, and W. D. Blair, "A two-stage Kalman estimator for state estimation in the presence of random bias and for tracking maneuvering targets," in Proceedings of the 30th IEEE Conference on Decision and Control, pp. 20592062, December 1991.

[29] J. Y. Keller and M. Darouach, "Optimal two-stage Kalman filter in the presence of random bias," Automatica, vol. 33, no. 9, pp. 1745-1748, 1997.

[30] M. Ignagni, "Optimal and suboptimal separate-bias Kalman estimators for a stochastic bias," IEEE Transactions on Automatic Control, vol. 45, no. 3, pp. 547-551, 2000.

[31] B. Xu and Z. Wang, "Biased bearings-only parameter estimation for bistatic system," Journal of Electronics, vol. 24, no. 3, pp. 326331, 2007.

[32] B. Xu, Z. Wu, and Z. Wang, "On the Cramér-Rao lower bound for biased bearings-only maneuvering target tracking," Signal Processing, vol. 87, no. 12, pp. 3175-3189, 2007.

[33] X. He and D. Zhou, "Robust $H$-infinity filtering for time-delay systems with missing measurements: a parameter-dependent approach," Journal of Control Theory and Applications, vol. 5, no. 4, pp. 336-344, 2007.

[34] S. Sun, "Optimal linear filters for discrete-time systems with randomly delayed and lost measurements with/without time stamps," IEEE Transactions on Automatic Control, vol. 58, no. 6, pp. 1551-1556, 2013. 


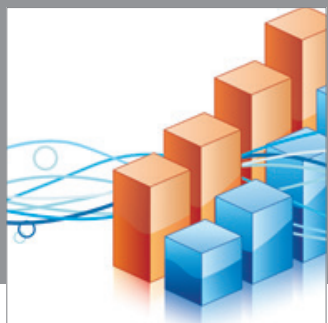

Advances in

Operations Research

mansans

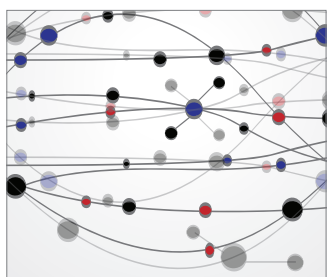

The Scientific World Journal
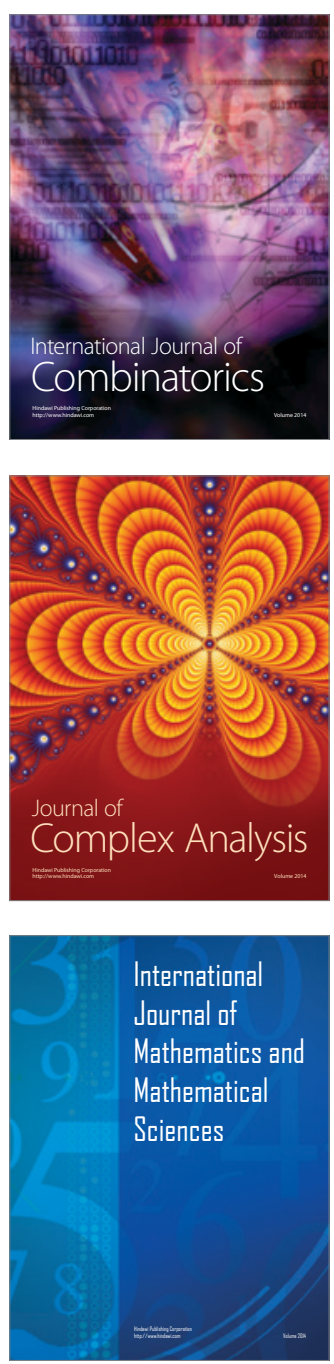
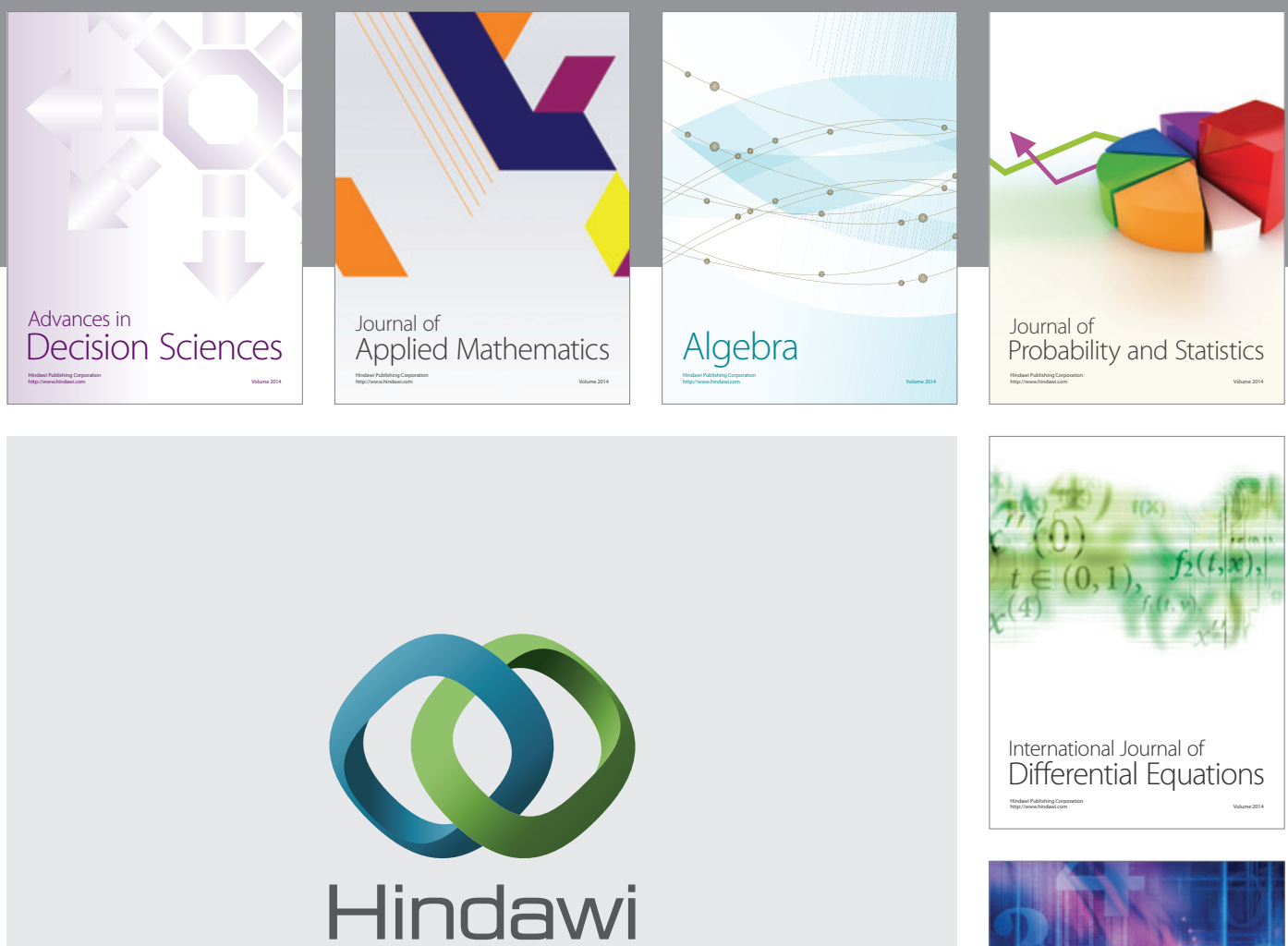

Submit your manuscripts at http://www.hindawi.com
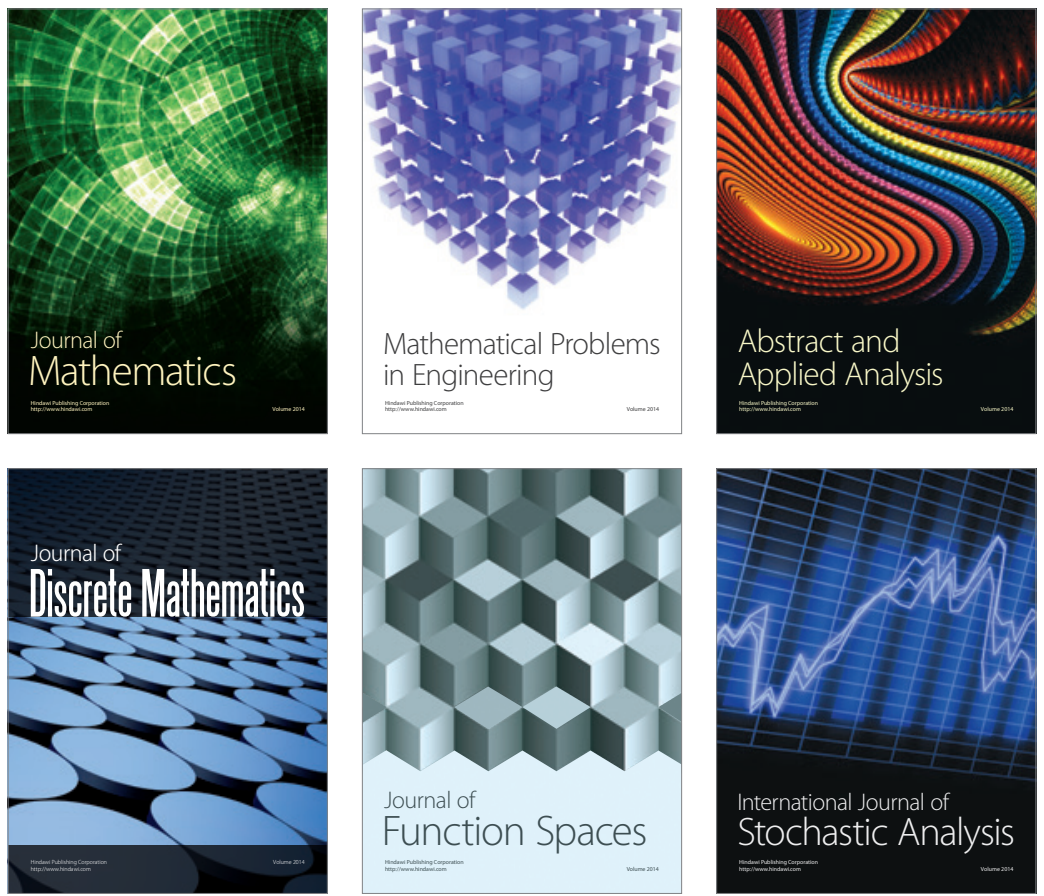

Journal of

Function Spaces

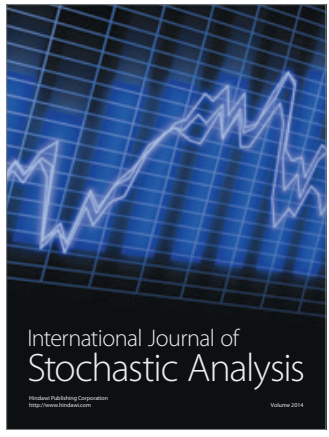

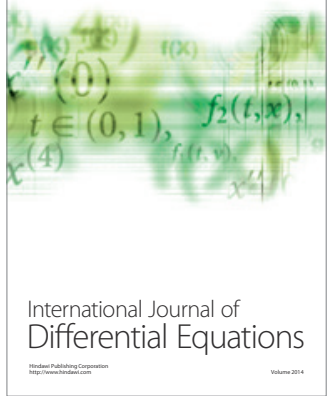
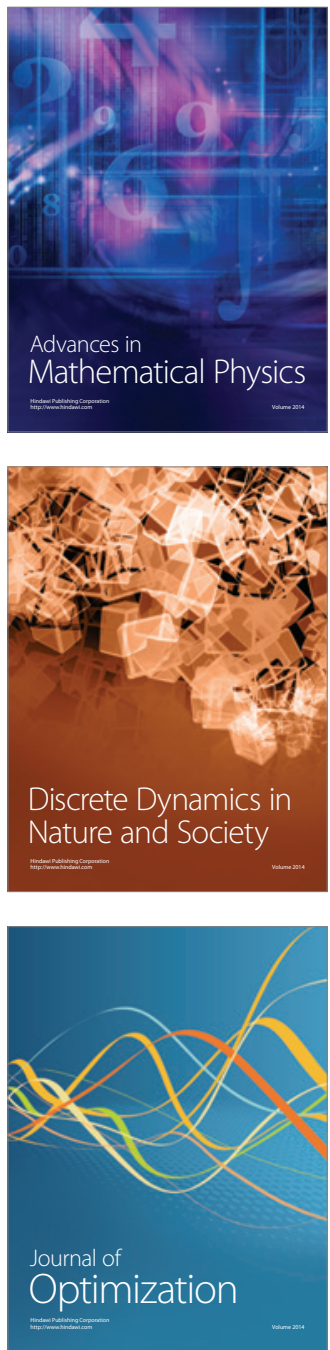